\title{
Optimal eddy viscosity for resolvent-based models of coherent structures in turbulent jets
}

\author{
Ethan Pickering ${ }^{1}$, Georgios Rigas ${ }^{1}$, Oliver T. Schmidt ${ }^{2}$, Denis Sipp ${ }^{3}$, \\ Tim Colonius ${ }^{1}$ \\ ${ }^{1}$ Division of Engineering and Applied Science, California Institute of Technology, Pasadena, \\ CA 91125, USA \\ ${ }^{2}$ Mechanical and Aerospace Engineering, University of California, San Diego, La Jolla, CA \\ 92093, USA \\ ${ }^{3}$ ONERA - The French Aerospace Lab, 92190 Meudon, France
}

(Received xx; revised xx; accepted xx)

Response modes computed via linear resolvent analysis of the turbulent mean-flow field have been shown to qualitatively capture characteristics of the observed turbulent coherent structures in both wall-bounded and free shear flows. To make such models predictive, the nonlinear forcing term must be closed either by including a self-consistent set of triadic interactions or through turbulence modeling. For the latter, several investigators have proposed using the mean-field eddy viscosity acting linearly on the fluctuation field. In this study, a data-driven approach is taken to quantitatively improve linear resolvent models by deducing an optimal eddy-viscosity field that maximizes the projection of the dominant resolvent mode to the energy-optimal coherent structure educed using spectral proper orthogonal decomposition (SPOD) of data from high-fidelity simulations. We use large-eddy simulation databases for round isothermal jets at subsonic, transonic, and supersonic conditions and show that the optimal eddy viscosity substantially improves the alignment between resolvent and SPOD modes, reaching over $90 \%$ alignment at those frequencies where the jet exhibits a low-rank response. We then consider a fixed model for the eddy viscosity and show that with the calibration of a single constant, the results are generally close to the optimal one. In particular, the use of a standard ReynoldsAveraged-Navier-Stokes (RANS) eddy-viscosity resolvent model, with a single scaling coefficient, provides substantial agreement between SPOD and resolvent modes for three turbulent jets and across the most energetic wavenumbers and frequencies.

\section{Introduction}

Resolvent analysis (also known as input/output analysis) determines a volumetric distribution of forcing in the frequency domain that gives rise, when acting in a timeinvariant flow, to the most amplified linear response, typically in terms of its total kinetic energy. It is an important tool in stability and transition analysis (Trefethen et al. 1993; Farrell \& Ioannou 1993; Schmid et al. 2002; Jovanović \& Bamieh 2005), and has more recently been proposed as a reduced-order model of coherent structures in fully developed turbulence (McKeon \& Sharma 2010; Hwang \& Cossu 2010b). In the latter context, resolvent analysis can be derived by partitioning of the Navier-Stokes equations into terms that are linear and nonlinear with respect to perturbations to the turbulent mean flow. Such a rearrangement of the equations is exact, and the equations may be explored without recourse to any further modeling. With varying degrees of formality, similar approaches were proposed in the past (Malkus 1956; Michalke 1971; Crighton \& Gaster 1976; Butler \& Farrell 1992), but increases in computer power that speed up the 
singular value decomposition (SVD) of the linear operator using direct LU decomposition (multi-frontal algorithms for sparse systems) have allowed a detailed characterization of the resolvent spectrum in several turbulent, canonical wall-bounded (Hwang \& Cossu 2010a,b; McKeon \& Sharma 2010; Sharma \& McKeon 2013; Moarref et al. 2013) and free shear flows (Jeun et al. 2016; Schmidt et al. 2018).

In particular, at those frequencies where the dominant singular value is significantly larger than the subdominant singular values, the highest-gain modes are qualitatively predictive of the structure, but not necessarily amplitude, of coherent modes educed from corresponding experimental and computational datasets (Schmidt et al. 2018). To construct a predictive reduced-order model, it is necessary to include the nonlinear interactions comprising the forcing terms. Out of many possibilities, one approach, arguably the simplest, is to use an eddy viscosity. Concepts underlying the triple decomposition can motivate this (Reynolds \& Tiederman 1967; Reynolds \& Hussain 1972), which identifies the Reynolds stresses as acting on the coherent fluctuations from both coherent and incoherent fluctuations, though the phase average used to define the coherent part of the field is ambiguous in unforced turbulent flows. Several authors have taken this perspective throughout the wall-bounded turbulence literature (Del Alamo \& Jimenez 2006; Cossu et al. 2009; Pujals et al. 2009; Hwang \& Cossu 2010a,b; Hwang 2016; Vadarevu et al. 2019) either through implementing the Cess (1958) eddy-viscosity model or by estimation of the eddy-viscosity field via the Reynolds stresses and mean shear rate of strain. Similarly, global stability analyses have applied eddy-viscosity models to identify and/or control forced or self-sustained resonances in transitional and turbulent flows (Crouch et al. 2007; Meliga et al. 2012; Mettot et al. 2014; Sartor et al. 2014; Semeraro et al. 2016; Tammisola \& Juniper 2016; Rukes et al. 2016; Oberleithner et al. 2014). These studies implemented eddy-viscosity on an ad hoc basis, citing improved qualitative agreement or improved integrated energy-densities.

In a more quantitative sense, eddy-viscosity enhanced linearized models have also proven useful for assimilating known data to reconstruct observed energy spectra and mean-flow quantities. Using eddy-viscosity enhanced linear models, Moarref \& Jovanović (2012) showed that a data-driven, white-in-time forcing method could reproduce the DNS-based turbulent energy spectrum, while Illingworth et al. (2018), also using a data-driven linear modeling approach, matched DNS energy spectra using time-resolved velocity measurements. Pickering et al. (2020) used an eddy-viscosity enhanced resolvent model to reconstruct the large-eddy simulation (LES) acoustic field of transonic and supersonic turbulent jets at a significantly lower rank when compared to their non-eddyviscosity enhanced computations. Not only does the inclusion of eddy-viscosity models increase the ability of linear models to reconstruct DNS/LES statistics, but they are an ideal quantity for coupling linear models to RANS equations, particularly for predicting the effect of active open-loop control (Moarref \& Jovanović 2012). Other approaches have implemented eddy-viscosity fields to develop self-consistent models, such as Yim et al. (2019), who recently coupled a harmonically forced, quasi-linear resolvent analysis with RANS equations, citing eddy viscosity as a necessary link between the coherent and incoherent perturbation dynamics.

Although the utility of eddy-viscosity enhanced linear models for turbulent modeling and control has become increasingly apparent, a question that has received less attention is: what is the quantitative effect of eddy-viscosity on modeling turbulent structures? For stationary turbulence, one data-driven answer is quantitatively assessing resolvent modes against one (or more) spectral proper orthogonal decomposition (SPOD) modes and their amplitudes educed from computational or experimental datasets. SPOD of turbulent flow data provides a set of orthogonal space-time correlated modes, ranked by energy, that 
optimally describe the turbulent flow statistics (Towne et al. 2018). This provides a useful benchmark for reduced-order turbulent models, but there is a deeper connection. Towne et al. (2018) showed that if resolvent forcing is spatially uncorrelated, then the resolvent response modes must be identical to the SPOD modes. As noted above, the agreement between SPOD (true response) and resolvent modes (theoretical response) to date has only been qualitative (with few exceptions, i.e. Morra et al. (2019)); quantitative discrepancies between the modes implies correlation between the resolvent forcings. A colored-noise process may represent these correlations and some recent approaches have sought to determine its covariance structure based on matching (partially) observed statistics (Zare et al. 2017; Towne et al. 2020).

Morra et al. (2019) applied a similar line of thinking by including an eddy viscosity in their resolvent analysis of turbulent channel flow, showing that the resulting resolvent modes were better predictive of the SPOD modes educed from high-fidelity simulation data than resolvent analysis using only molecular viscosity. We extend this approach to turbulent jets, but consider a more general framework. Informally speaking, we want to see "how much color" can be removed from the forcing by including an eddy-viscosity model in the resolvent operator. In this approach, an ideal model would render the forcing uncorrelated, meaning that the resolvent and SPOD modes coincide. We therefore define a data-informed variational problem that seeks an optimal eddy-viscosity field that maximizes the projection of the first SPOD mode on the first resolvent mode. We then show that we can achieve nearly optimal projections using standard eddy-viscosity models, including one directly inferred from a corresponding Reynolds-Average NavierStokes (RANS) simulation.

From a more general perspective, this work may also be linked to the building of dataaugmented turbulence models (Duraisamy et al. 2019). Here, we specifically target the modeling of unsteady features (Wang et al. 2018; Maulik et al. 2019) and the optimal eddy-viscosity fields obtained, at each frequency-wavenumber pair, and this study can be seen as a field-inversion step (also based on variational data-assimilation methods, Foures et al. (2014); Parish \& Duraisamy (2016)) to assist machine learning techniques for generating eddy-viscosity models from mean-flow quantities.

The paper is organized as follows. In $\S 2$ we outline the governing equations, resolvent analysis, and SPOD. In $\S 3$ we discuss the optimization framework developed to align SPOD and resolvent modes, and the specific eddy-viscosity models examined. $\S 4$ provides the resulting resolvent mode shapes found via the four eddy-viscosity models methods and $\S 5$ analyzes the associated optimal eddy-viscosity fields. In $\S 6$ we show a favorable impact of the eddy-viscosity models on the subdominant resolvent modes and then conclude the analysis in $\S 7$ by assessing the sensitivity of the RANS eddy-viscosity model. In this final section, we ultimately find a frequency independent RANS eddy-viscosity field that performs well for three turbulent jets (i.e. subsonic, transonic, and supersonic) and over the most energetic frequencies $(S t \in[0.05-1])$ and azimuthal wavenumbers $(m \in[0,5])$.

\section{Methods}

The LES database, resolvent analysis, and SPOD were described in Schmidt et al. (2018) and Towne et al. (2018). For brevity, we only recall the main details here.

\subsection{Large Eddy Simulation database}

The flow solver Charles was used to compute the LES databases, including subsonic (Mach 0.4), transonic (Mach 0.9), and supersonic (Mach 1.5) cases; Brès et al. (2017) contains the details on the numerical method, meshing, and subgrid-models. Experiments 


$\begin{array}{cccccccc}\text { case } & M_{j} & R e_{j} & \frac{p_{0}}{p_{\infty}} & \frac{T_{0}}{T_{\infty}} & n_{\text {cells }} & \Delta t a_{\infty} / D & \Delta S t \\ \text { subsonic } & 0.4 & 4.5 \times 10^{5} & 1.117 & 1.03 & 15.9 \times 10^{6} & 0.2 & 0.049 \\ \text { transonic } & 0.9 & 1.01 \times 10^{6} & 1.7 & 1.15 & 15.9 \times 10^{6} & 0.2 & 0.022 \\ \text { supersonic } & 1.5 & 1.76 \times 10^{6} & 3.67 & 1.45 & 31 \times 10^{6} & 0.1 & 0.026\end{array}$

Table 1: Parameters, sampling rate, and frequency resolution for the LES.

conducted at PPRIME Institute, Poitiers, France were used to validate the Mach 0.4 and 0.9 jets (Brès et al. 2018). Table 1 provides a summary of parameters for the three jets considered. Parameters include the Reynolds number based on diameter $R e_{j}=\rho_{j} U_{j} D / \mu_{j}$ (where subscript $j$ specifies the value at the centerline of the jet nozzle exit, $\rho$ is density, $\mu$ is viscosity) and the Mach number, $M_{j}=U_{j} / a_{j}$, where $a_{j}$ is the speed of sound. The simulated $M_{j}=0.4$ jet corresponds to the experiments in Cavalieri et al. (2013); Jaunet et al. (2017); Nogueira et al. (2019) with the same nozzle geometry and similar boundarylayer properties at the nozzle exit. Throughout the manuscript, reported results are nondimensionalized by the mean jet velocity $U_{j}$, jet diameter $D$, and dynamic pressure $\rho_{j} U_{j}^{2}$. We report frequencies in Strouhal number, $S t=f D / U_{j}$, where $f$ is the frequency.

Each database consists of 10,000 snapshots separated by $\Delta t a_{\infty} / D$, where $a_{\infty}$ is the ambient speed of sound, and interpolated onto a structured cylindrical grid $x, r, \theta \in$ $[0,30] \times[0,6] \times[0,2 \pi]$, where $x, r, \theta$ are streamwise, radial, and azimuthal coordinates, respectively. Variables are reported by the vector

$$
\boldsymbol{q}=\left[\rho, u_{x}, u_{r}, u_{\theta}, T\right]^{T},
$$

where $u_{x}, u_{r}, u_{\theta}$ are the three velocity components, and a standard Reynolds decomposition separates the vector into mean, $\overline{\boldsymbol{q}}$, and fluctuating, $\boldsymbol{q}^{\prime}$, components

$$
\boldsymbol{q}(x, r, \theta, t)=\overline{\boldsymbol{q}}(x, r, \theta)+\boldsymbol{q}^{\prime}(x, r, \theta, t) .
$$

\subsection{Resolvent analysis}

We start with the nonlinear flow equations of the form

$$
\frac{\partial \boldsymbol{q}}{\partial t}=\boldsymbol{F}(\boldsymbol{q})
$$

where $\boldsymbol{F}$ is the time-independent compressible Navier-Stokes operator (plus continuity and energy). Substituting equation (2.2) for $\boldsymbol{q}$ and separating terms linear in state perturbations, $\boldsymbol{q}^{\prime}$, to the left-hand side gives

$$
\frac{\partial \boldsymbol{q}^{\prime}}{\partial t}-\boldsymbol{A}(\overline{\boldsymbol{q}}) \boldsymbol{q}^{\prime}=\boldsymbol{f}\left(\overline{\boldsymbol{q}}, \boldsymbol{q}^{\prime}\right)
$$

where

$$
\boldsymbol{A}(\overline{\boldsymbol{q}})=\frac{\partial \boldsymbol{F}}{\partial \boldsymbol{q}}(\overline{\boldsymbol{q}})
$$

is the linearized flow operator and $\boldsymbol{f}$ contains the nonlinear terms, and any additional external inputs (e.g. environmental noise or perturbations at the boundary).

For the round, statistically-stationary turbulent jets we consider, equation (2.4) is Fourier transformed both temporally and azimuthally to the compact expression

$$
\left(i \omega \mathbf{I}-\mathbf{A}_{m}\right) \boldsymbol{q}_{m, \omega}=\boldsymbol{f}_{m, \omega} .
$$

We can then rewrite equation (2.6) by defining the resolvent operator, $\boldsymbol{R}_{\omega, m}=(i \omega \boldsymbol{I}-$ 
$\left.\boldsymbol{A}_{m}\right)^{-1}$

$$
\boldsymbol{q}_{m, \omega}=\boldsymbol{R}_{m, \omega} \boldsymbol{f}_{m, \omega},
$$

and introduce the compressible energy norm (Chu 1965),

$$
\left\langle\boldsymbol{q}_{1}, \boldsymbol{q}_{2}\right\rangle_{E}=\iiint \boldsymbol{q}_{1}^{*} \operatorname{diag}\left(\frac{\bar{T}}{\gamma \bar{\rho} M^{2}}, \bar{\rho}, \bar{\rho}, \bar{\rho}, \frac{\bar{\rho}}{\gamma(\gamma-1) \bar{T} M^{2}}\right) \boldsymbol{q}_{2} r \mathrm{~d} r \mathrm{~d} x \mathrm{~d} \theta,
$$

via the matrix $\boldsymbol{W}$ to the forcing and response, where $\boldsymbol{W}=\boldsymbol{W}_{f}=\boldsymbol{W}_{q}$. Taking the singular value decomposition of the resolvent operator gives

$$
\boldsymbol{R}_{m, \omega}=\boldsymbol{U}_{m, \omega} \boldsymbol{\Sigma}_{m, \omega} \boldsymbol{V}_{m, \omega}^{*} \boldsymbol{W},
$$

where the diagonal matrix $\boldsymbol{\Sigma}_{m, \omega}$ contain the ranked gains and the columns of $\boldsymbol{U}_{m, \omega}$ and $\boldsymbol{V}_{m, \omega}$ contain the response and forcing modes, respectively, that are orthonormal in the energy norm, equation (2.8):

$$
\boldsymbol{U}_{m, \omega}^{*} \boldsymbol{W} \boldsymbol{U}_{m, \omega}=\boldsymbol{V}_{m, \omega}^{*} \boldsymbol{W} \boldsymbol{V}_{m, \omega}=\boldsymbol{I} .
$$

For the resolvent analysis presented here, just as in Schmidt et al. (2018), the above equations are discretized in the streamwise and radial directions with fourth-order summation by parts finite differences (Mattsson \& Nordström 2004), while the polar singularity is treated as in Mohseni \& Colonius (2000) and non-reflecting boundary conditions are implemented at the domain boundaries.

\subsection{Spectral Proper Orthogonal Decomposition}

SPOD, similar to space-only proper orthogonal decomposition (POD) and originally shown by Lumley $(1967,1970)$, determines an optimal (i.e. in terms of energy) set of orthogonal modes to describe a dataset, but unlike space-only POD, produces modes that express both spatial and temporal correlation in the data. Like dynamic mode decomposition (DMD), each mode is associated with a unique frequency, but are naturally ranked by energy and, through appropriate averaging, best account for statistical variability in turbulent flows (Towne et al. 2018). Thus, the associated SPOD modes provide the ideal measurement tool for which to assess modes computed via resolvent analysis.

Decomposing the LES database $\boldsymbol{Q}$, where $\boldsymbol{Q}$ represents the temporal ensemble of perturbations $\left(\boldsymbol{q}^{\prime}\right)$ found by applying the standard Reynolds decomposition, in the azimuthal and temporal dimensions via the discrete Fourier transform gives the decomposed data matrices, $\hat{\boldsymbol{Q}}_{m, \omega}$. The cross-spectral density tensor at a given frequency $\omega=2 \pi S t$ and azimuthal wavenumber $m$ is then given by

$$
\mathbf{S}_{m, \omega}=\hat{\boldsymbol{Q}}_{m, \omega} \hat{\boldsymbol{Q}}_{m, \omega}^{*}
$$

and the SPOD eigenvalue problem presented by Lumley $(1967,1970)$ can be solved

$$
\mathbf{S}_{m, \omega} \mathbf{W} \boldsymbol{\Psi}_{m, \omega}=\boldsymbol{\Psi}_{m, \omega} \boldsymbol{\Lambda}_{m, \omega} .
$$

The SPOD modes form the columns of $\boldsymbol{\Psi}_{m, \omega}$ and are ranked by the diagonal matrix of eigenvalues $\boldsymbol{\Lambda}_{m, \omega}=\operatorname{diag}\left(\lambda_{1}, \lambda_{2}, \ldots, \lambda_{N}\right)$. The modes are orthonormal in the norm $\langle\cdot, \cdot\rangle_{E}$, and satisfy $\boldsymbol{\Psi}_{m, \omega}^{*} \boldsymbol{W} \boldsymbol{\Psi}_{m, \omega}=\boldsymbol{I}$. As a consequence, the cross-spectral density tensor may be expanded as,

$$
\mathbf{S}_{m, \omega}=\boldsymbol{\Psi}_{m, \omega} \boldsymbol{\Lambda}_{m, \omega} \boldsymbol{\Psi}_{m, \omega}^{*} .
$$

In this study, all SPOD computations are done implementing a Hanning window with realization sizes of 256 snapshots and $50 \%$ overlap, resulting in 78 independent realizations. 
To avoid ambiguity in referring to computed SPOD and resolvent modes, we use the following notation for the rest of the manuscript. First, all computed modes subscripts $m, \omega$ are dropped, but referenced when necessary in the text. Second, $\boldsymbol{\psi}_{n}$ represents the $n$-th most energetic SPOD mode, while $\boldsymbol{v}_{n}$ and $\boldsymbol{u}_{n}$ denote the resolvent forcing and response, respectively, that provide the $n$-th largest linear-amplification gain between $\boldsymbol{v}_{n}$ and $\boldsymbol{u}_{n}$. Finally, when referring to specific components of each mode, such as streamwise velocity, the notation $\psi_{1}: u_{x}$ is used.

\subsection{Theoretical connection of resolvent and SPOD}

Recently, multiple authors have reported the theoretical connection between resolvent analysis and SPOD (Towne et al. 2015; Semeraro et al. 2016; Towne et al. 2018; Schmidt et al. 2018). The connection is between the response and forcing cross-spectral density (CSD) matrices via the resolvent operator. Assuming any pair of wavenumbers/frequencies, we define the CSD for the response $\boldsymbol{S}_{\boldsymbol{q} \boldsymbol{q}}=\mathbb{E}\left[\boldsymbol{q} \boldsymbol{q}^{*}\right]$ and forcing $\boldsymbol{S}_{\boldsymbol{f f}}=$ $\mathbb{E}\left[\boldsymbol{f} \boldsymbol{f}^{*}\right]$, with $\mathbb{E}[\cdot]$ as the expectation operator. Applying the expectation operator to equation (2.7) multiplied by its complex conjugate gives

$$
\boldsymbol{S}_{\boldsymbol{q q}}=\mathbb{E}\left[\boldsymbol{q \boldsymbol { q } ^ { * }}\right]=\mathbb{E}\left[\boldsymbol{R} \boldsymbol{f} \boldsymbol{f}^{*} \boldsymbol{R}^{*}\right]=\boldsymbol{R} \boldsymbol{S}_{\boldsymbol{f f}} \boldsymbol{R}^{*} .
$$

The above equation directly relates the cross-spectral densities of forcing and response terms with the resolvent operator. We may then expand the forcing CSD, $\boldsymbol{S}_{\boldsymbol{f} \boldsymbol{f}}=$ $\boldsymbol{\Psi}_{\boldsymbol{f}} \boldsymbol{\Lambda}_{\boldsymbol{f} f} \boldsymbol{\Psi}_{\boldsymbol{f}}^{*}$ with $\boldsymbol{\Psi}_{\boldsymbol{f}}^{*} \boldsymbol{W} \boldsymbol{\Psi}_{\boldsymbol{f}}=\boldsymbol{I}$, and using the decomposition of the resolvent operator, yields

$$
S_{q q}=U \boldsymbol{\Sigma} \boldsymbol{V}^{*} \boldsymbol{W} \boldsymbol{\Psi}_{f} \boldsymbol{\Lambda}_{f f} \Psi_{f}^{*} \boldsymbol{W} \boldsymbol{V} \boldsymbol{\Sigma} \boldsymbol{U}^{*}
$$

Defining the projection of the forcing SPOD basis in the resolvent forcing basis, $\boldsymbol{\Psi}_{f}=$ $\boldsymbol{V} \boldsymbol{\beta}$, we have $\boldsymbol{\beta}=\boldsymbol{V}^{*} \boldsymbol{W} \boldsymbol{\Psi}_{\boldsymbol{f}}$ and $\boldsymbol{\beta}^{*} \boldsymbol{\beta}=\boldsymbol{I}$, so that

$$
\boldsymbol{S}_{\boldsymbol{q q}}=\boldsymbol{U} \boldsymbol{\Sigma} \boldsymbol{\beta} \boldsymbol{\Lambda}_{\boldsymbol{f f}} \boldsymbol{\beta}^{*} \boldsymbol{\Sigma} \boldsymbol{U}^{*}=\boldsymbol{\Psi} \boldsymbol{\Lambda} \boldsymbol{\Psi}^{*} .
$$

This expression may then be reduced using a variety of assumptions on the properties of the forcing basis.

Here we consider the case of spatially-uncorrelated forcing and do so by linking the form of the SPOD forcing modes to those found by resolvent analysis. Assuming response modes of SPOD and resolvent are aligned, $\boldsymbol{\Psi}=\boldsymbol{U}$, as is imposed in this study via an eddy-viscosity turbulence model, then equation (2.6) requires $\boldsymbol{\Psi}_{\boldsymbol{f}}=\boldsymbol{V}$. This reduces the projection of the forcing modes to $\boldsymbol{\beta}=\boldsymbol{I}$, and equation (2.16) to

$$
\begin{aligned}
\boldsymbol{S}_{\boldsymbol{q q}} & =\boldsymbol{U} \boldsymbol{\Sigma} \boldsymbol{\Lambda}_{\boldsymbol{f f}} \boldsymbol{\Sigma} \boldsymbol{U}^{*} \\
& =\boldsymbol{\Psi} \boldsymbol{\Lambda} \boldsymbol{\Psi}^{*} .
\end{aligned}
$$

The above establishes two relationships between SPOD and resolvent analysis under spatially uncorrelated forcing (Towne et al. 2018). The first is that the resolvent modes will be identical to the set of SPOD modes. Second, the equations provide a relation between the eigenvalue spectra and the forcing energy as $\boldsymbol{\Lambda}_{\boldsymbol{f f}}=\boldsymbol{\Sigma}^{-1} \boldsymbol{\Lambda} \boldsymbol{\Sigma}^{-1}$. Here $\boldsymbol{\Lambda}_{\boldsymbol{f f}}$ is a diagonal matrix, per the definition of the uncorrelated case, but these values vary along the diagonal as there is no assumption on the forcing magnitude experienced by each mode. We discuss the implications of this result and its relationship to various turbulent amplification mechanisms in $\S 7$.

In the next section, we present various physical models designed to impose the uncorrelated condition by maximizing the alignment of dominant singular vectors with dominant SPOD modes. 


\begin{tabular}{lcccr} 
Turbulence model & $\boldsymbol{\mu}_{T}$ form & Optimal parameter & LES data used & Abbreviation \\
\hline Baseline* & $1 / R e_{T}=3 \times 10^{4}$ & - & - & Baseline \\
Optimal field & $\boldsymbol{\mu}_{T}(\boldsymbol{x})$ & $\boldsymbol{\mu}_{T}(\boldsymbol{x})$ & $\boldsymbol{\Psi}$ & Opt. $\boldsymbol{\mu}_{T}$ \\
Mean-flow consistent & $c \boldsymbol{\mu}_{T}(\boldsymbol{x})$ & $c$ & $\overline{\boldsymbol{q}}$ & Mean $\boldsymbol{\mu}_{T}$ \\
RANS & $c \bar{\rho} C_{\mu} k^{2} / \epsilon$ & $c$ & - & RANS $\boldsymbol{\mu}_{T}$ \\
Turbulent Re & $1 / \mathrm{Re}_{T}$ & $1 / \mathrm{Re}_{T}$ & - & $\operatorname{Re}_{T, O p t}$
\end{tabular}

Table 2: Turbulence models investigated in this study. The baseline* case refers to the results of Schmidt et al. (2018).

\section{Models considered}

Here we seek to construct a resolvent model that is predictive of observed turbulent structure by finding a turbulence closure whose inclusion in the forward linear operator, $\boldsymbol{L}=\left(i \omega \boldsymbol{I}-\boldsymbol{A}_{m}\right)$ (i.e. $\left.\boldsymbol{L}=\boldsymbol{R}^{-1}\right)$, optimizes the alignment between the dominant resolvent and SPOD modes. We consider four models for the eddy viscosity in detail. The first directly optimizes the eddy-viscosity field to maximize alignment between the dominant resolvent and SPOD modes. The second model establishes an eddy viscosity associated with the mean flow from LES by imposing a Boussinessq ansatz. The third model uses an independently-computed eddy-viscosity field from a RANS $k-\epsilon$ model. Finally, we consider a simple, constant eddy-viscosity model based upon a turbulent Reynolds number. Table 2 summarizes the various models investigated.

Parenthetically, within the optimization framework we can consider any turbulence closure based on mean-flow quantities. A further example is given in appendix A, where we consider a linear damping model recently proposed for resolvent analysis of unstable base flows by Yeh \& Taira (2019).

For all models, we supplement the molecular viscosity, $\mu_{j}$, with a turbulent one, $\boldsymbol{\mu}_{T}(\mathbf{x})$, via the following terms to the right-hand side of the continuity, momentum, and energy equations (Appendix B includes the full equations), respectively,

$$
\left[\begin{array}{c}
0 \\
\nabla \cdot\left[\boldsymbol{\mu}_{T}\left((\nabla \boldsymbol{u})+(\nabla \boldsymbol{u})^{T}-\frac{2}{3} \Theta \mathbb{I}\right)\right] \\
\frac{\boldsymbol{\mu}_{T}}{(\gamma-1) M_{j}^{2} P r_{\infty}} \nabla^{2} T+\gamma M_{j}^{2} \boldsymbol{\mu}_{T}\left[\frac{1}{2}\left\{(\nabla \boldsymbol{u})+(\nabla \boldsymbol{u})^{T}\right\}:\left\{(\nabla \boldsymbol{u})+(\nabla \boldsymbol{u})^{T}\right\}-\frac{2}{3} \Theta^{2}\right]
\end{array}\right],
$$

where $\operatorname{Pr}_{\infty}=0.7, \Theta=\nabla \cdot \boldsymbol{u}$ is the dilatation, and $\mathbb{I}$ is the second order identity tensor. The eddy-viscosity term, $\boldsymbol{\mu}_{T}$, is nondimensionalized by $\rho_{j}, U_{j}, D$ and in sum with the molecular viscosity presents an effective viscosity $\boldsymbol{\mu}_{\text {eff }}=\boldsymbol{\mu}_{T}+1 / R e_{j}$.

In the exact rearrangement of the full equations (2.6), $\boldsymbol{f}$ inherently includes the modeled terms described in equation (3.1). Thus in our approach $\boldsymbol{f}$ is decomposed into (now) linear terms associated with the eddy viscosity, which we denote as $\boldsymbol{L}_{T} \boldsymbol{q}$ (i.e. $\boldsymbol{L}_{T}$ is the linearized (around $\overline{\boldsymbol{q}}$ ) operator associated to the discretized version of equation (3.1)), and a remainder forcing (still potentially nonlinear), $\boldsymbol{g}=\boldsymbol{f}-\boldsymbol{L}_{T} \boldsymbol{q}$. Then equation (2.6) becomes

$$
\begin{array}{r}
\boldsymbol{L} \boldsymbol{q}=\boldsymbol{L}_{T} \boldsymbol{q}+\boldsymbol{g} \\
\boldsymbol{L}_{\boldsymbol{\mu}_{T}} \boldsymbol{q}=\boldsymbol{g}
\end{array}
$$


where $\boldsymbol{L}_{\boldsymbol{\mu}_{T}}=\boldsymbol{L}-\boldsymbol{L}_{T}$ is the forward linear operator including the chosen turbulence model.

\subsection{Optimal eddy-viscosity field}

Here we optimize for the entire spatial eddy-viscosity field, computed independently for each frequency and azimuthal mode. The purpose of such an optimization is to determine an upper bound to which any eddy-viscosity model can align the leading resolvent and SPOD modes. Thus we take our optimization parameter as $\boldsymbol{\mu}_{T}$ so that the number of optimization variables is equal to the number of grid points in the $x-r$ plane.

We construct the optimization with the goal of discerning an optimal modification to the linear operator that maximized the projection coefficients between SPOD and resolvent modes. To do so, we prescribe a constrained optimization problem subject to the governing equations, the resolvent analysis, a normalization constraint, and constraints associated with the assumed eddy-viscosity ansatz for modifying the operator. We couple all components of the optimization through a Lagrangian functional and take variations with respect to each parameter to solve for the stationary point, or maximum.

To construct the Lagrangian functional we return to the forward equation (2.6) and

substitute $\boldsymbol{L}$ with $\boldsymbol{L}_{\boldsymbol{\mu}_{T}}$, the modified operator, for which we highlight four turbulence models in the following subsections. A singular value / singular vector $\left(\boldsymbol{v}_{1}, \boldsymbol{u}_{1}, \sigma_{1}\right)$ as defined in (2.9) is also a solution of both

$$
\begin{aligned}
\boldsymbol{v}_{1} & =\boldsymbol{L}_{\boldsymbol{\mu}_{T}} \boldsymbol{u}_{1} \\
\boldsymbol{W} \boldsymbol{u}_{1} & =\sigma_{1}^{2} \boldsymbol{L}_{\boldsymbol{\mu}_{T}}^{*} \boldsymbol{W} \boldsymbol{v}_{1} .
\end{aligned}
$$

and fulfills the normalization constraint,

$$
\left\langle\boldsymbol{u}_{1}, \boldsymbol{u}_{1}\right\rangle_{E}=\boldsymbol{u}_{1}^{*} \boldsymbol{W} \boldsymbol{u}_{1}=1 .
$$

We then construct the cost function as

$$
\mathcal{J}=\boldsymbol{u}_{1}^{*} \boldsymbol{W} \boldsymbol{\psi}_{1} \boldsymbol{\psi}_{1}^{*} \boldsymbol{W} \boldsymbol{u}_{1}-l^{2} \boldsymbol{\mu}_{T}^{*} \boldsymbol{M} \boldsymbol{\mu}_{T},
$$

which seeks to maximize the square of the projection between the dominant SPOD mode, $\boldsymbol{\psi}_{1}$, and first resolvent mode, $\boldsymbol{u}_{1}$. By taking the squared absolute value of the projection, $\boldsymbol{u}_{1}^{*} \boldsymbol{W} \boldsymbol{\psi}_{1}$, we ensure that the cost is real. For brevity, we denote the outer product of the dominant SPOD mode as $\boldsymbol{\Psi}_{1}=\boldsymbol{\psi}_{1} \boldsymbol{\psi}_{1}^{*}=\boldsymbol{\Psi}_{1}^{*}$. The term $-l^{2} \boldsymbol{\mu}_{T}^{*} \boldsymbol{M} \boldsymbol{\mu}_{T}$ penalizes values of $\boldsymbol{\mu}_{T}$ that are unnecessary to achieve alignment (high values of $\boldsymbol{\mu}_{T}$ diminish the value of $\mathcal{J})$, with $M$ representing the cylindrical quadrature weights of the grid. The value of $l^{2}$ should be chosen high enough to remove the values of $\boldsymbol{\mu}_{T}$ in insensitive regions but also sufficiently small not to substantially affect the alignment (Hansen \& O'Leary 1993). It is also straightforward to consider multiple resolvent / SPOD modes by considering a (weighted if desired) sum of the squared alignment terms.

We now construct the full Lagrangian functional combining the cost function (3.7), forward equation (3.4), resolvent eigenvalue problem (3.5), and the constraint (3.6) to give,

$$
\begin{aligned}
\mathcal{L} & =\boldsymbol{u}_{1}^{*} \boldsymbol{W} \boldsymbol{\psi}_{1} \boldsymbol{\psi}_{1}^{*} \boldsymbol{W} \boldsymbol{u}_{1}-l^{2} \boldsymbol{\mu}_{T}^{*} \boldsymbol{M} \boldsymbol{\mu}_{T} \\
& -\tilde{\boldsymbol{u}}_{1}^{*}\left(\boldsymbol{L}_{\boldsymbol{\mu}_{T}} \boldsymbol{u}_{1}-\boldsymbol{v}_{1}\right)-\tilde{\boldsymbol{v}}_{1}^{*}\left(\boldsymbol{W} \boldsymbol{u}_{1}-\sigma_{1}^{2} \boldsymbol{L}_{\boldsymbol{\mu}_{T}}^{*} \boldsymbol{W} \boldsymbol{v}_{1}\right)-\tilde{\sigma}_{1}\left(\boldsymbol{u}_{1}^{*} \boldsymbol{W} \boldsymbol{u}_{1}-1\right)+\text { c.c. }
\end{aligned}
$$

The newly acquired terms, $\left(\tilde{\boldsymbol{u}}_{1}, \tilde{\boldsymbol{v}}_{1}, \tilde{\sigma}_{1}\right)$, in the Lagrangian functional are Lagrangian multipliers and c.c. are the complex conjugates to ensure real values. This results in a functional that depends on six variables,

$$
\mathcal{L}\left(\left[\boldsymbol{u}_{1}, \boldsymbol{v}_{1}, \sigma_{1}\right],\left[\tilde{\boldsymbol{u}}_{1}, \tilde{\boldsymbol{v}}_{1}, \tilde{\sigma}_{1}\right]\right) .
$$



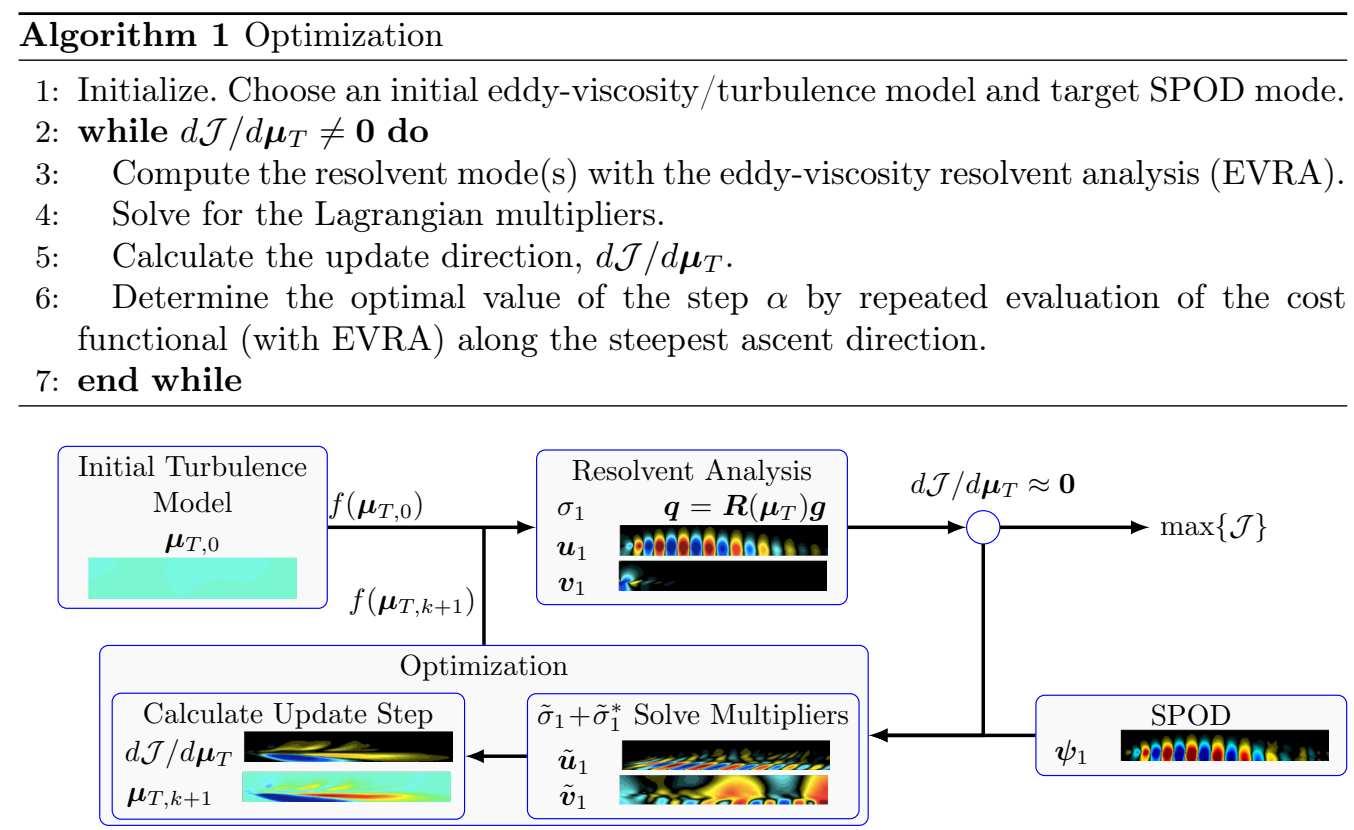

Figure 1: Schematic of the optimization framework for determining the optimal eddyviscosity field that maximizes the alignment between computed resolvent modes, $\boldsymbol{u}_{1}$, and educed SPOD modes, $\boldsymbol{\psi}_{\mathbf{1}}$. Included graphics are from implementation of the full-field eddy-viscosity model at $S t=0.6, m=0$, and $M_{j}=0.4$.

Stationarity of the Lagrangian with respect to the multipliers yields the state equations, which are by definition satisfied, while stationarity with respect to the state variables yields:

$$
\begin{array}{ll}
\frac{\partial \mathcal{L}}{\partial \boldsymbol{u}_{1}} \delta \boldsymbol{u}_{1}=\left(2 \boldsymbol{W} \boldsymbol{\Psi}_{1} \boldsymbol{W} \boldsymbol{u}_{1}-\boldsymbol{L}_{\boldsymbol{\mu}_{T}}^{*} \tilde{\boldsymbol{u}}_{1}-\boldsymbol{W} \tilde{\boldsymbol{v}}_{1}-\left(\tilde{\sigma}_{1}+\tilde{\sigma}_{1}^{*}\right) \boldsymbol{W} \boldsymbol{u}_{1}\right)^{*} \delta \boldsymbol{u}_{1} & =0 \\
\frac{\partial \mathcal{L}}{\partial \boldsymbol{v}_{1}} \delta \boldsymbol{v}_{1}=\left(\tilde{\boldsymbol{u}}_{1}+\sigma_{1}^{2} \boldsymbol{W} \boldsymbol{L}_{\boldsymbol{\mu}_{T}} \tilde{\boldsymbol{v}}_{1}\right)^{*} \delta \boldsymbol{v}_{1} & =0 \\
\frac{\partial \mathcal{L}}{\partial \sigma_{1}} \delta \sigma_{1}=\left(\tilde{\boldsymbol{v}}_{1}^{*} \boldsymbol{L}_{\boldsymbol{\mu}_{T}}^{*} \boldsymbol{W} \boldsymbol{v}_{1}\right)^{*} \delta \sigma_{1} & =0,
\end{array}
$$

and the condition in the last equation may be simplified into $\tilde{\boldsymbol{v}}_{1}^{*} \boldsymbol{L}_{\boldsymbol{\mu}_{T}}^{*} \boldsymbol{W} \boldsymbol{v}_{1}=\tilde{\boldsymbol{v}}_{1}^{*} \boldsymbol{W} \boldsymbol{u}_{1}$ using equation (3.5). The stationary point is subsequently met by constructing the following matrix and solving for the Lagrangian multipliers:

$$
\left[\begin{array}{ccc}
-\boldsymbol{L}_{\boldsymbol{\mu}_{T}}^{*} & -\boldsymbol{W} & -\boldsymbol{W} \boldsymbol{u}_{1} \\
\boldsymbol{W}^{-1} & \boldsymbol{L}_{\boldsymbol{\mu}_{T}} \sigma_{1}^{2} & 0 \\
0 & \boldsymbol{u}_{1}^{*} \boldsymbol{W} & 0
\end{array}\right]\left[\begin{array}{c}
\tilde{\boldsymbol{u}}_{1} \\
\tilde{\boldsymbol{v}}_{1} \\
\tilde{\sigma}_{1}+\tilde{\sigma}_{1}^{*}
\end{array}\right]=\left[\begin{array}{c}
-2 \boldsymbol{W} \boldsymbol{\Psi}_{1} \boldsymbol{W} \boldsymbol{u}_{1} \\
0 \\
0
\end{array}\right]
$$

The upper left $2 \times 2$ block is degenerate due to the state equations (3.4) and (3.5) (the couple $\left(\tilde{\boldsymbol{u}}_{1}=\boldsymbol{W} \boldsymbol{v}_{1}, \tilde{\boldsymbol{v}}_{1}=-\sigma_{1}^{-2} \boldsymbol{u}_{1}\right)$ is in the null-space of this block). The third column and line, therefore, regularizes this system. Combining the 3 equations, it is easy to show that $\tilde{\sigma}_{1}+\tilde{\sigma}_{1}^{*}=2 \boldsymbol{u}_{1}^{*} \boldsymbol{W} \boldsymbol{\Psi}_{1} \boldsymbol{W} \boldsymbol{u}_{1}$, which is indeed a positive real value.

To perform the optimization, a final variation is taken with respect to a prescribed parameter, or set of parameters, $\boldsymbol{\mu}_{T}$ (which may be a scalar or vector quantity), that affect the forward operator $\boldsymbol{L}_{\boldsymbol{\mu}_{T}}$. The variation with respect to these parameters provides 
the direction of gradient ascent for the optimization parameter

$$
\begin{aligned}
\frac{\partial \mathcal{L}}{\partial \boldsymbol{\mu}_{T}} \delta \boldsymbol{\mu}_{T} & =-\tilde{\boldsymbol{u}}_{1}^{*}\left(\frac{\partial \boldsymbol{L}_{\boldsymbol{\mu}_{T}}}{\partial \boldsymbol{\mu}_{T}} \delta \boldsymbol{\mu}_{T}\right) \boldsymbol{u}_{1}+\sigma_{1}^{2} \tilde{\boldsymbol{v}}_{1}^{*}\left(\frac{\partial \boldsymbol{L}_{\boldsymbol{\mu}_{T}}^{*}}{\partial \boldsymbol{\mu}_{T}} \delta \boldsymbol{\mu}_{T}\right) \boldsymbol{W} \boldsymbol{v}_{1}-2 l^{2} \boldsymbol{\mu}_{T}^{*} \boldsymbol{M} \delta \boldsymbol{\mu}_{T}+c . c \\
& =\left(\frac{d \mathcal{J}}{d \boldsymbol{\mu}_{T}}\right)^{*} \boldsymbol{M} \delta \boldsymbol{\mu}_{T} .
\end{aligned}
$$

The gradient at the $k^{\text {th }}$ grid point is:

$$
\left.\frac{d \mathcal{J}}{d \boldsymbol{\mu}_{T}}\right|_{k}=\boldsymbol{M}_{k m}^{-1}\left(-\boldsymbol{u}_{1, j}^{*} \boldsymbol{L}_{m, i j}^{*} \tilde{\boldsymbol{u}}_{1, i}+\sigma_{1}^{2} \boldsymbol{W}_{l j} \boldsymbol{v}_{1, l}^{*} \boldsymbol{L}_{m, j i} \tilde{\boldsymbol{v}}_{1, i}\right)-2 l^{2} \boldsymbol{\mu}_{T, k}+c . c,
$$

where $\boldsymbol{L}_{m, i j}=\lim _{\epsilon \rightarrow 0} \frac{\boldsymbol{L}_{\boldsymbol{\mu}_{T}+\epsilon \delta \boldsymbol{\mu}_{m}, i j}-\boldsymbol{L}_{\boldsymbol{\mu}_{T}, i j}}{\epsilon}, \delta \boldsymbol{\mu}_{m}$ being a null vector except at the $m^{t h}$ position where it is equal to 1 . This tensor can be obtained either by automatic differentiation of $\boldsymbol{L}_{\boldsymbol{\mu}_{T}}$ with respect to $\boldsymbol{\mu}_{T}$ or by finite differences. Full storage of such tensors is not an issue if finite differences, finite volumes, or finite elements are used for the spatial discretization as the resulting tensors will be extremely sparse.

The updated parameter is then:

$$
\boldsymbol{\mu}_{T}^{(k+1)}=\boldsymbol{\mu}_{T}^{(k)}+\alpha \frac{d \mathcal{J}}{d \boldsymbol{\mu}_{T}},
$$

where $k$ is the iteration number and $\alpha$ is a step size determined through a root finding algorithm or a line search. If multiple SPOD / resolvent modes are considered for the optimization then one has to solve eq. (3.13) for each couple $\left[\boldsymbol{\Psi}_{i},\left(\boldsymbol{v}_{i}, \boldsymbol{u}_{i}, \sigma_{i}\right)\right]$ and the total gradient $\frac{d \mathcal{J}}{d \boldsymbol{\mu}_{T}}$ is the sum of each individual gradient, while the line search for $\alpha$ has to be performed with the full cost functional. Figure 1 presents a schematic of the above optimization framework, including graphical examples from the optimal eddy-viscosity field case at $S t=0.6, m=0$, and $M_{j}=0.4$.

When $\boldsymbol{\mu}_{T}$ involves many degrees of freedom, the topology of the cost function may be very complex, and our optimizer may return a local rather than global maximum. In the case where the optimization step creates a region of negative eddy-viscosity, that region is set to zero so that only the molecular viscosity is present. For this case, a complete assessment of the sensitivity of initial conditions or demonstration of a global maximum are intractable, but the relative insensitivity of the results to initial guesses and the fact that no other considered method outperforms the full optimization (shown later in figure 4) provide some confidence in the robustness of the maxima achieved. Apart from cases that varied the initial guesses, the results presented here used the optimal constant eddy-viscosity field results as the initial condition for the full-field optimization.

\subsection{Mean-flow consistent eddy-viscosity model}

For many experimental and numerical datasets, including the LES databases used here, an eddy-viscosity field is absent. We circumvent this issue by finding the eddy-viscosity field that minimizes the error to which the mean flow satisfies the (zero frequency) eddyviscosity equations (3.1). That is, at zero frequency and zero azimuthal wavenumber, we seek an eddy viscosity that minimizes a residual $\bar{f}$ given by

$$
\boldsymbol{L}_{\boldsymbol{\mu}_{T}} \overline{\boldsymbol{q}}=\overline{\boldsymbol{f}} .
$$

Thus we define the cost function:

$$
\mathcal{J}=-\overline{\boldsymbol{f}}^{*} \boldsymbol{W} \overline{\boldsymbol{f}}
$$


and develop a Lagrangian with the forward equation as the only constraint to give

$$
\mathcal{L}=-\overline{\boldsymbol{f}}^{*} \boldsymbol{W} \overline{\boldsymbol{f}}-\tilde{\boldsymbol{u}}^{*}(\boldsymbol{L} \overline{\boldsymbol{q}}-\overline{\boldsymbol{f}}) .
$$

The variations with respect to the residual are

$$
\frac{\partial \mathcal{L}}{\partial \overline{\boldsymbol{f}}} \delta \overline{\boldsymbol{f}}=(-2 \boldsymbol{W} \overline{\boldsymbol{f}}+\tilde{\boldsymbol{u}})^{*} \delta \overline{\boldsymbol{f}}=0,
$$

which may be directly solved for the multipliers as,

$$
\tilde{\boldsymbol{u}}=-2 \boldsymbol{W} \overline{\boldsymbol{f}} .
$$

Then taking variations with respect to the eddy-viscosity field gives,

$$
\frac{\partial \mathcal{L}}{\partial \boldsymbol{\mu}_{T}} \delta \boldsymbol{\mu}_{T}=-2(\boldsymbol{W} \overline{\boldsymbol{f}})^{*}\left(\frac{\partial \boldsymbol{L}}{\partial \boldsymbol{\mu}_{T}} \delta \boldsymbol{\mu}_{T}\right) \overline{\boldsymbol{q}} .
$$

Similarly to eq. (3.16), we obtain the update step:

$$
\left.\frac{d \mathcal{J}}{d \boldsymbol{\mu}_{T}}\right|_{k}=-2 \boldsymbol{M}_{k m}^{-1} \overline{\boldsymbol{q}}_{j} \boldsymbol{L}_{m, i j} \boldsymbol{W}_{i l} \overline{\boldsymbol{f}}_{l},
$$

and the field is found by performing a line search and iterating until the solution converges. These steps are described in detail in the preceding subsection. Figure 2 (a) provides the eddy-viscosity field that optimally minimizes the residual of the meanflow solution. The associated residual field for this model was able to reduce errors to approximately $10 \%$ of the original residual field, with the exception where the shear layer is thin near the nozzle. The thin shear-layer region improved by only $\approx 50 \%$, but as will be shown, modes in this region are generally less sensitive to the eddy-viscosity field.

We refer to this model as the mean-flow consistent eddy-viscosity model. The field is optimally tuned at each frequency by introducing the scaling constant, $c$,

$$
\boldsymbol{\mu}_{T}(S t)=c(S t) \boldsymbol{\mu}_{T, M e a n} .
$$

\subsection{Reynolds-Averaged Navier-Stokes}

RANS solutions are computed for each of the turbulent jet cases to assess the applicability of the associated eddy-viscosity field for resolvent analysis. For simplicity, RANS solutions were performed in Fluent. The 2D axisymmetric grid extended 40 diameters in the streamwise directions and 20 diameters in the radial direction with grid spacing mirroring that of the interpolated LES grid scaled to be four times finer, giving $3 \times 10^{5}$ grid points. The inlet boundary condition was set to the base-flow profile from the LES simulations and the standard 2-equation $k-\epsilon$ model was used for turbulence modeling. Coefficients used for the model are variants of those suggested by Thies \& Tam (1996), where $C_{\mu}=0.0874, C_{\epsilon 1}=1.4, C_{\epsilon 2}=2.02, \sigma_{K}=0.324, \sigma_{\epsilon}=0.377$ and the turbulent Prandtl number $\operatorname{Pr}_{T}=0.422$. However, the standard $\kappa-\epsilon$ model provided in ANSYS does not incorporate the Pope (1978) and Sarkar et al. (1991) correction terms used in Thies \& Tam (1996), requiring a calibration of the mean-flow quantities by introducing a scaling constant $a$ to $C_{\mu}=0.0874 / a, \sigma_{K}=0.324 / a$, and $\sigma_{\epsilon}=0.377 / a$.

RANS base-flow quantities closely matched those of the LES for each of the three turbulent jets using values for $a$ of $1.2,1.3$, and 1.575, for $M_{j}=0.4,0.9$, and 1.5 , respectively. While tuning of the constant $a$ and using LES as the truth is not in the spirit of obtaining a universal RANS model, we did so here to give the RANS-generated eddy-viscosity field the best chance at correctly modeling the resolvent forcing. However, in the framework of turbulence modelling, diminishing the value of $C_{\mu}$, that we impose 
(a)

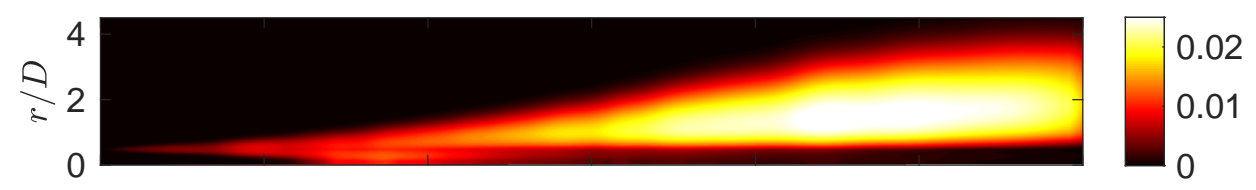

(b)

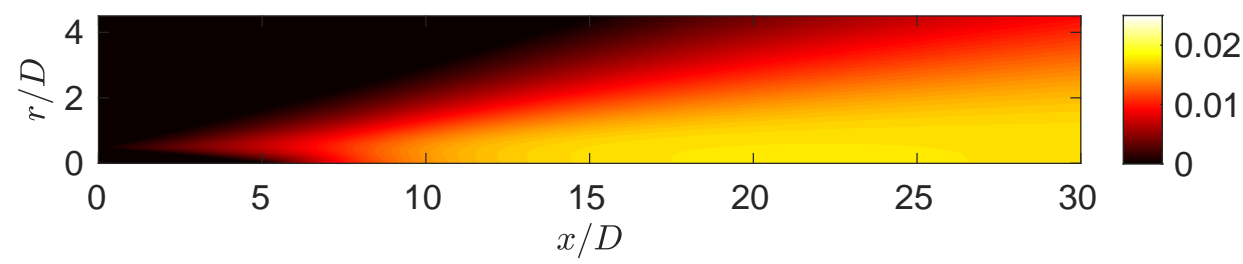

Figure 2: (a) Mean-flow consistent eddy-viscosity model computed at zero frequency and azimuthal wavenumber. (b) Eddy-viscosity field computed via a RANS simulation for the $M_{j}=0.4$ jet, $c=1$.

(a)

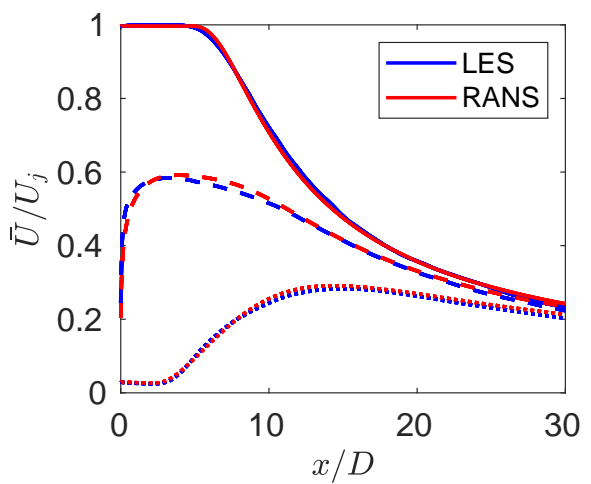

(b)

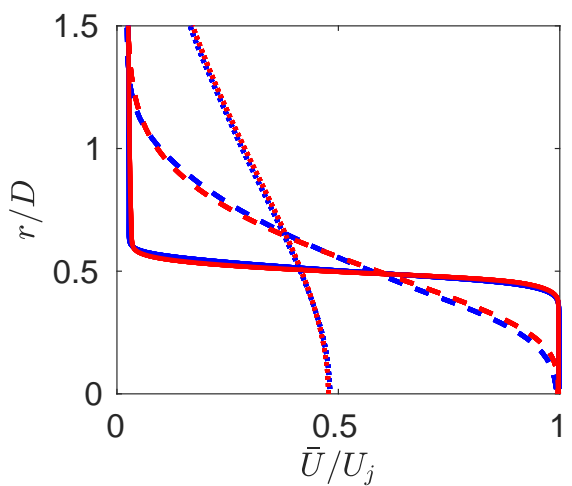

Figure 3: Mean-flow profiles of both the $M_{j}=0.4$ LES and RANS, where the RANS simulation was tuned to best match the LES mean flow. (a) presents the streamwise mean velocity at three radial locations, $r / D=-0.25,---0.5, \cdots \cdot 1$, versus streamwise distance from the nozzle, while (b) gives the streamwise mean velocity at three streamwise locations, $x / D=-0.5,--5, \cdots . .10$, versus radial distance.

with tuning $a$, is a classical means to allow RANS models to exhibit unsteady features in the case of turbulent flows exhibiting coherent structures at precise frequency, such as cylinder or airfoils at high angle of attack (Hoarau 2002; Hoarau et al. 2003; Deprés 2003). Yet, there is not a universal value for $C_{\mu}$, which may, depending on the coherent structures at play, range between 0.02 and 0.06 (for a reference value of $C_{\mu}=0.0874$ ). For a full assessment of the accuracy of RANS predictions for turbulent jets we refer the reader to Thies \& Tam (1996); Georgiadis et al. (2006), and the robustness and accuracy of the RANS closure on resolvent results is left for future work.

Figure 2 (b) presents the RANS-predicted eddy-viscosity field for the $M_{j}=0.4$ jet and figure 3 shows near identical agreement with the mean LES streamwise flow. Similar agreement was observed in radial velocity, density, and turbulent kinetic energy, and all these quantities showed approximately the same agreement for the $M_{j}=0.9$ and 1.5 
jets; these results are not shown for brevity. For determination of the optimal RANSbased eddy-viscosity field at each frequency we take the computed eddy-viscosity fields and permit the field to be altered by a scaling constant $c$ (just as in the preceding subsection), such that

$$
\boldsymbol{\mu}_{T}(S t)=c(S t) \boldsymbol{\mu}_{T, R A N S}=c(S t) \bar{\rho} C_{\mu} \frac{k^{2}}{\epsilon} .
$$

\subsection{Constant eddy-viscosity field}

Finally, we consider a simple, constant eddy viscosity $\boldsymbol{\mu}_{T}=1 / R e_{T}$. We primarily investigate this model due to its use in many turbulent jet studies that used a Reynolds number based either upon the molecular viscosity (Jeun et al. 2016; Lesshafft et al. 2019), on the order of $10^{5}-10^{6}$, or through an effective turbulent viscosity (Garnaud et al. 2013; Schmidt et al. 2018), on the order of $10^{3}-10^{4}$. These, quite different, choices inevitably provided discrepancies in amplification gains and mode shapes across each study, particularly at low frequencies (i.e. $S t<0.3$ for $m=0$ ) - showing that the Reynolds stresses have a substantial impact on resolvent analyses of turbulent jets. Here, the optimal $\operatorname{Re}_{T}$ is found at each frequency and azimuthal mode number by a line search. We also consider the baseline case for which $R e_{T}=3 \times 10^{4}$. This value of $\operatorname{Re}_{T}=3 \times 10^{4}$ was used in a previous comparison of resolvent and SPOD modes for the same jets considered here in Schmidt et al. (2018).

\section{Optimal SPOD and resolvent mode alignment}

In this section, we present modes predicted by the various eddy-viscosity resolvent analyses (EVRA) models presented in the previous section. We focus on the axisymmetric disturbances, $m=0$, for the $M_{j}=0.4$ jet, and report results for other azimuthal modes and jet Mach numbers in section 7 . We performed optimizations over the frequency range $S t=0.05-1$, resulting in the alignment coefficients displayed in figure 4 . Recall that the alignment coefficient is defined as $\left|\boldsymbol{\psi}_{1}^{*} \boldsymbol{W} \boldsymbol{u}_{1}\right|$. This metric not only represents how similar the spatial structures, represented as complex eigenfunctions, are between the dominant resolvent and SPOD modes, but also measures the similarity in distribution of energy amongst the five state variables. A value of 1 is perfect agreement, giving both identical agreement in structure and distribution of energy in the state variables. Typically, in this metric, values of approximately 0.4 or greater are indicative of qualitative agreement, whereas values less than 0.4 show little visual similarity.

Figure 4 shows that throughout the frequency range considered, the alignments improve considerably from the baseline case (constant eddy viscosity with $R e_{T}=3 \times 10^{4}$ ). Generally, the alignment is best for $S t>0.3$, which corresponds to the frequencies where the jet has a strong, low-rank Kelvin-Helmholtz response (Schmidt et al. 2018), and for which even the baseline case gives reasonable ( $>75 \%$ alignment) results. The improvement in alignment is most dramatic at low frequencies. The optimal eddyviscosity field provides the best alignment (which is at least suggestive that we achieved a global maximum). At these low frequencies, $S t<0.3$, the non-optimized resolvent spectrum for the $M_{j}=0.4$ jet is dominated by Orr-type modes (Schmidt et al. 2018), a viscous, non-modal instability mechanism sensitive to Reynolds number, with rapidly increasing amplification as Reynolds number increases. Interestingly, the other eddyviscosity models produce alignments close to the optimal eddy-viscosity field. The constant eddy-viscosity is nearly optimal at lower frequencies (Orr-type modes), whereas the RANS and optimal mean-flow models are more nearly optimal at higher ones. We stress 


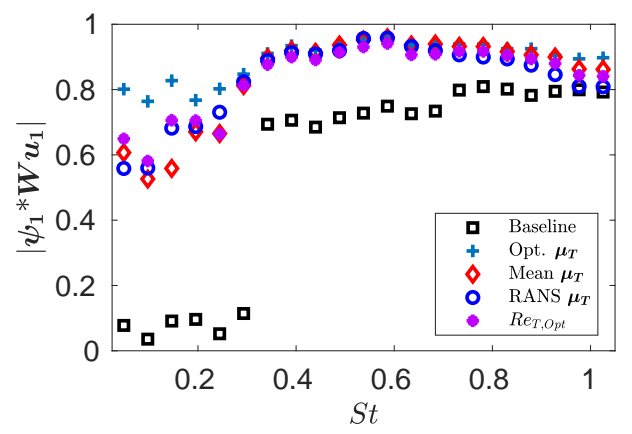

Figure 4: Optimal alignments for all methods investigated including the baseline case, $R e_{T}=3 \times 10^{4}$.
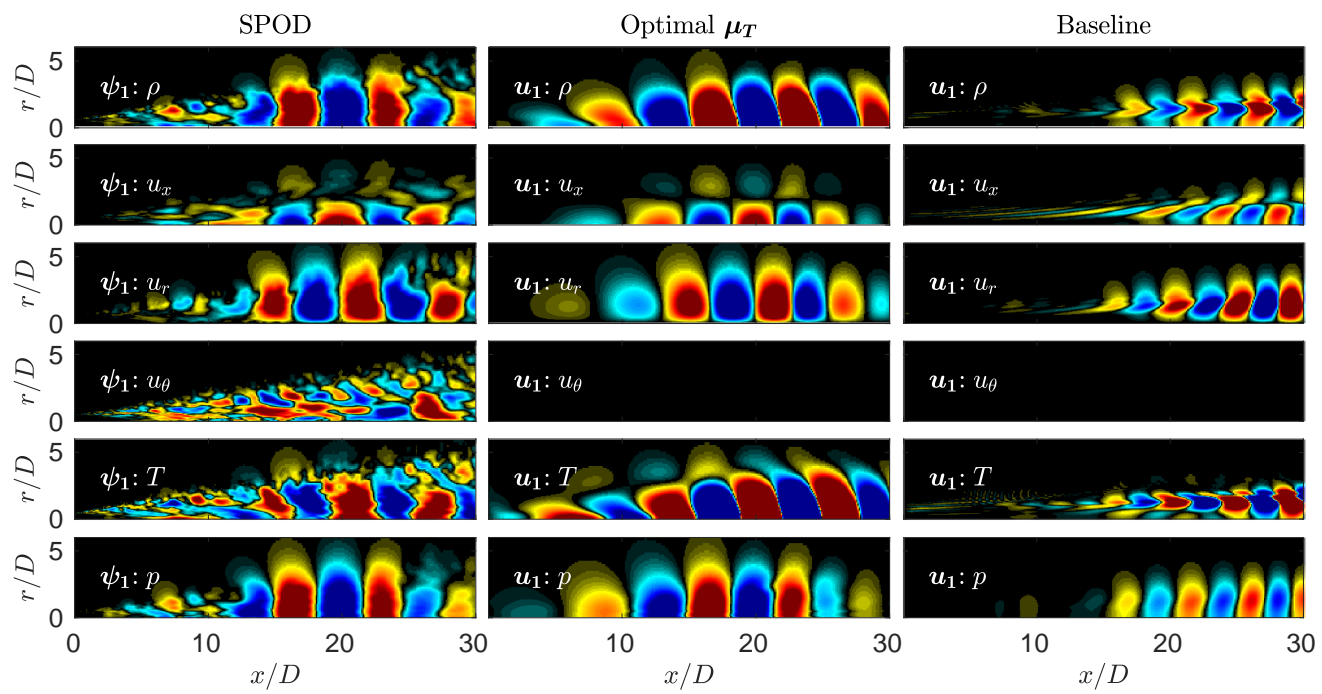

Figure 5: Real component of the fluctuating response state variables, $\boldsymbol{q}^{\prime}=$ $\left[\rho, u_{x}, u_{r}, u_{\theta}, T\right]$, and pressure, $p$, at $S t=0.05, m=0$. The columns display SPOD $\left(\boldsymbol{\psi}_{1}\right)$, optimal eddy viscosity $\left(\boldsymbol{u}_{1}\right)$, and baseline $\left(\boldsymbol{u}_{1}\right)$ modes from left to right, respectively. Contours $(\square)$ are given by $\pm 0.5\left\|\psi_{1}: \cdot\right\|_{\infty}$ of the SPOD mode, where - is the fluctuating variable in question (with $\left\|\boldsymbol{\psi}_{1}:\right\|_{\infty}$ values: $\left[\rho, u_{x}, u_{r}, u_{\theta}, T, p\right]=$ $\left.[2.8,198.6,46.0,37.2,1.2,10.4] \times 10^{-3}\right)$.

that in the optimal mean-flow, RANS, and constant $\boldsymbol{\mu}_{T}$ models, a different optimal value of the coefficient (i.e. $c$ and $R e_{T}$ ) has been used at each frequency. We differ a discussion of the sensitivity of these coefficients to $\S 7.1$.

Starting with the lowest frequency, $S t=0.05$, we now investigate the mode shapes associated with the improved resolvent alignments achieved with the optimized eddyviscosity models. Figure 5 displays the real part of the fluctuating field for all state variables for the dominant SPOD and resolvent modes, comparing resolvent results using both the optimal eddy-viscosity field and the baseline case with constant $R e_{T}=3 \times 10^{4}$. It 

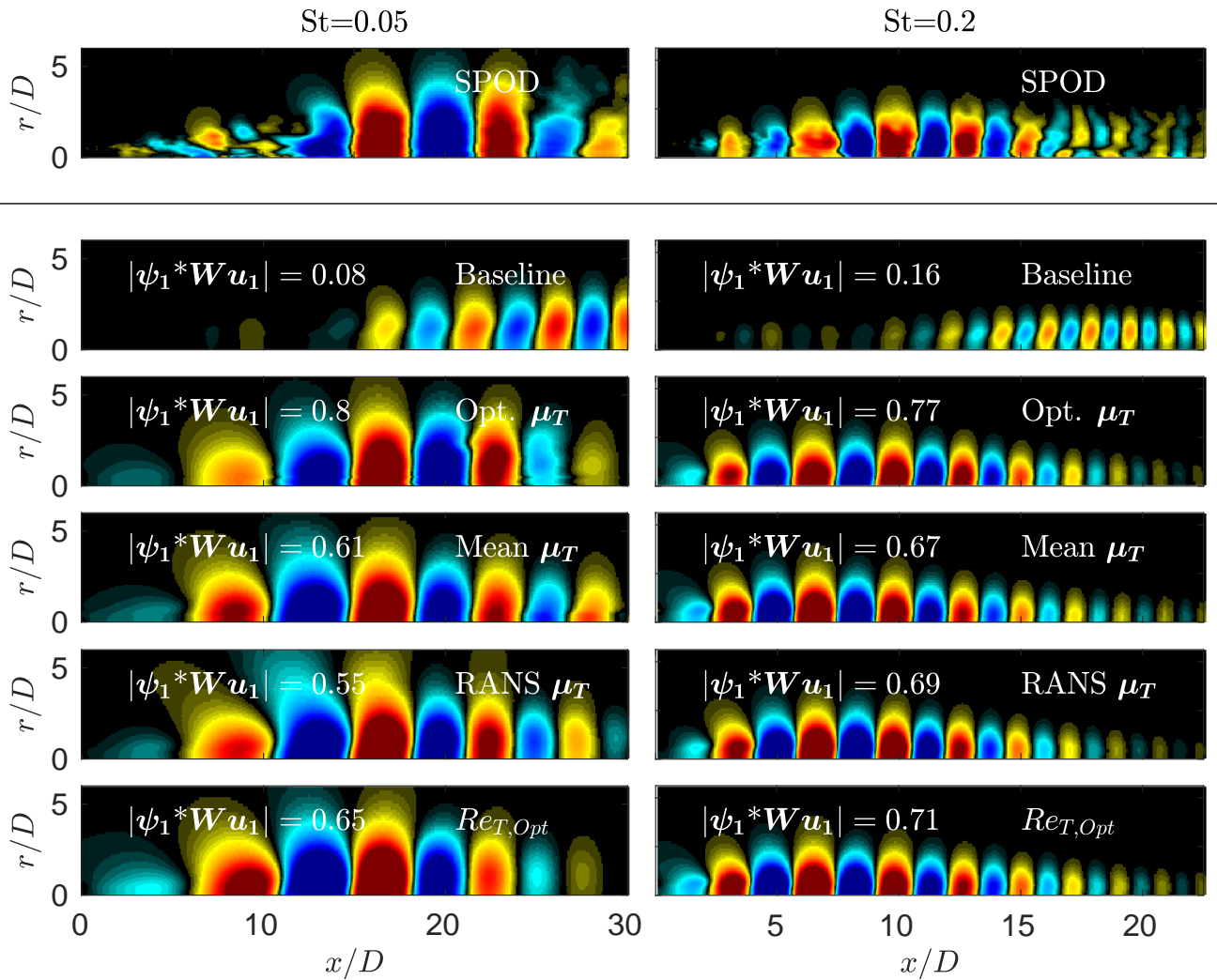

Figure 6: Real component of the response pressure fluctuations $\left(\boldsymbol{\square}, \pm 0.5\left\|\boldsymbol{\psi}_{1}: p\right\|_{\infty}\right)$ for $S t=0.05$ and $S t=0.2$ in the left and right columns, respectively. Row 1 presents the dominant SPOD mode for which the optimization seeks to match. The following rows present results for the baseline, optimal eddy-viscosity field, mean-flow consistent model, RANS eddy-viscosity model, and the optimal turbulent Reynolds number.

is immediately apparent that the resolvent mode with optimal-viscosity field can closely match the observed mode shapes from SPOD for all variables (including the correct distribution of energy), with the exception of $u_{\theta}^{\prime}$. By contrast, the baseline resolvent mode bears little resemblance to the SPOD modes for any of the variables.

Regarding $u_{\theta}^{\prime}$, it is exactly zero for the constant $\boldsymbol{\mu}_{T}$ field and very small for spatially varying $\boldsymbol{\mu}_{T}$ field (due to $m=0$ ). However, it is nonzero in the SPOD mode, and the reason for the discrepancy are statistical errors contained within SPOD modes, and which are strongest at low frequencies (because of the finite time series from the LES). Compared to the streamwise velocity, $u_{\theta}^{\prime}$ is about 5 times smaller in magnitude, and lacks the coherent wavepacket structure of the other variables. The corresponding $u_{\theta}$ contribution in the projection coefficient $\left|\boldsymbol{\psi}_{1}^{*} \boldsymbol{W} \boldsymbol{\psi}_{1}\right|$ is $\approx 0.08$, bounding the physical maximum of the optimization to $\left|\boldsymbol{\psi}_{1}^{*} \boldsymbol{W} \boldsymbol{u}_{1}\right| \leqslant 0.92$ without considering additional error in the other variables. These statistical errors are linked to the weak low-rank behavior with this frequency, and there is little separation between the dominant and subdominant modes (Schmidt et al. 2018). Indeed, the projection-coefficient value of 0.08 can be viewed as a kind of error bar on the alignments produced by the optimal eddy-viscosity field, as it is 

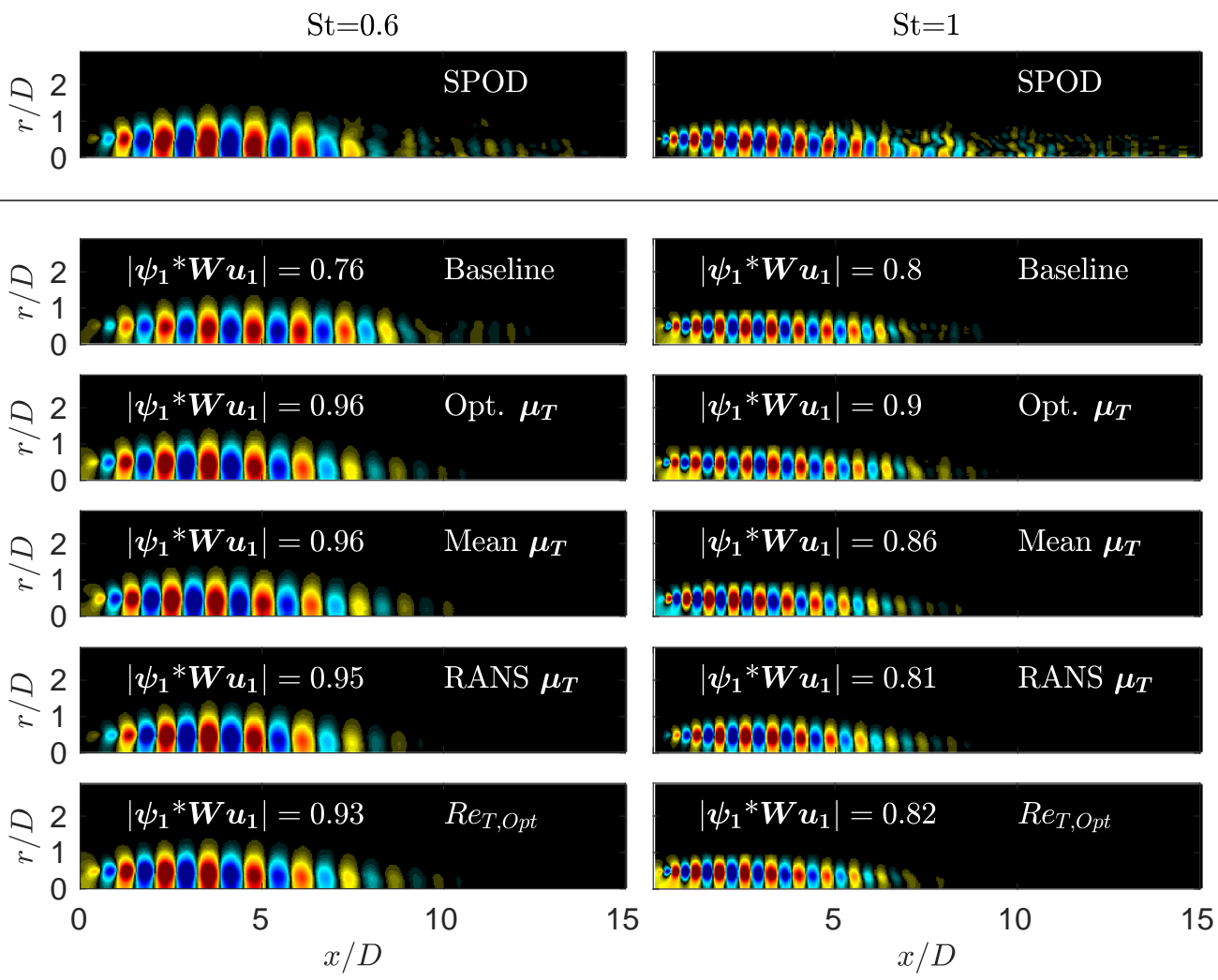

Figure 7: Real component of the response pressure fluctuations for $S t=0.6$ and $S t=1$ in the left and right columns, respectively. Rows present the equivalent methods as described in figure 6.

attempting to align to a mode shape that is (at this frequency) in error by as much as about $10 \%$. Likewise, it is gratifying that the optimization method was not "fooled" into trying to align with the statistical uncertainties associated with the SPOD mode.

The pressure field, a quantity of particular interest for jet noise, provides a relatively simple representative mode shape for each case. We proceed by visualizing only the fluctuating pressure component for the remainder of the study, however the projection coefficients, $\left|\boldsymbol{\psi}_{1}^{*} \boldsymbol{W} \boldsymbol{u}_{1}\right|$, account for the full state results. Further, for all response pressure modes presented, we see similar trends and improvements in all flow variables similar to figure 5 .

Figure 6 shows the pressure modes at two low frequencies, $S t=0.05$ and 0.2 , comparing now the results for all considered models. The top row shows the dominant SPOD mode from the LES, the second row gives the dominant resolvent mode for the baseline case, and the remaining rows provide four optimized models. At low frequency, the baseline resolvent analysis fails to capture the low frequency mode shapes. The optimized eddy-viscosity models, by contrast, have much better alignment with SPOD, increasing the projection coefficient by as much as 7 -fold, and displaying a wavepacket structure consistent with the SPOD mode. Recalling that at these low frequencies, $S t<0.3$, the baseline resolvent spectrum for a $M_{j}=0.4$ jet is dominated by Orr-type modes (Schmidt 

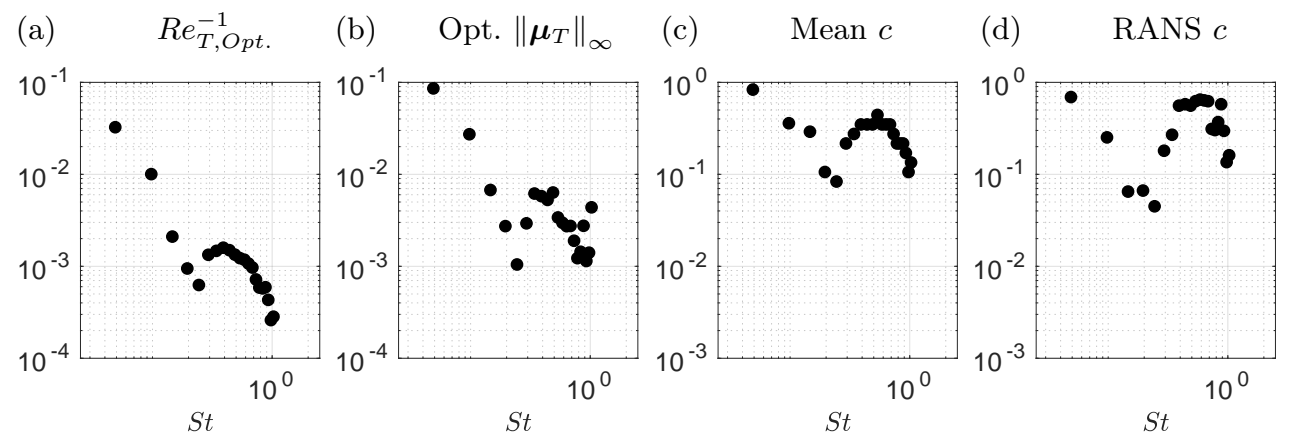

Figure 8: The optimal parameters across $S t \in[0.05-1]$ for (a) the optimal constant field $1 / R e_{T}$, (b) optimal eddy-viscosity field model, (c) the mean-flow consistent model, and (d) the optimal RANS model. The optimal eddy-viscosity field parameter shown is the maximum value of the field at each frequency, $\left\|\boldsymbol{\mu}_{T}\right\|_{\infty}$, while the latter two models present the optimal coefficient $c$. The associated alignments for each model/parameter are shown in figure 4 .

et al. 2018), we see that the eddy viscosity greatly attenuates these modes in favor of a Kelvin-Helmholtz (KH)-like response that peaks further upstream, and is consistent with what is observed in the SPOD modes.

Proceeding to higher frequencies, figure 7 displays the dominant fluctuating pressure modes for SPOD and the five resolvent methods for $S t=0.6$ and 1. Projection coefficients are already quite high for these frequencies, as was noted above, but are further increased with the eddy-viscosity models, reaching $96 \%$ for the optimal eddy viscosity. Here the differences in the mode shapes are subtle as the modes are generally shortened from the baseline case to better match the SPOD at both frequencies. At these higher frequencies, the jet response is a clear, low-rank $\mathrm{KH}$ wavepacket (a modal, inviscid stability mechanism) and it is thus unsurprising that the results are relatively insensitive to the precise eddy-viscosity model. However, the improved alignment is the result of a non-zero eddy-viscosity field, thus the turbulence model is still important.

For $S t=1$ the optimized projection coefficient is falling compared to the $S t=0.6$ case. This is again because of the emergence of Orr-modes with similar energy as the KH modes. By performing SPOD in limited domains near the nozzle exit, it becomes clear that the modal, low-rank $\mathrm{KH}$ response continues to much higher frequencies in the near nozzle region (Sasaki et al. 2017), but when considering the global response, these are always inferior in energy to the Orr response being excited and peaking further downstream.

\section{Analysis of the optimized eddy-viscosity fields}

The last section demonstrated that the optimized eddy-viscosity equipped resolvent modes resulted in a substantial alignment of the dominant resolvent and SPOD modes. In this section, we examine the optimal parameters associated with the eddy-viscosity fields to investigate how the eddy viscosity improved the alignment, and to look for clues about potential universalities in modeling coefficients. 

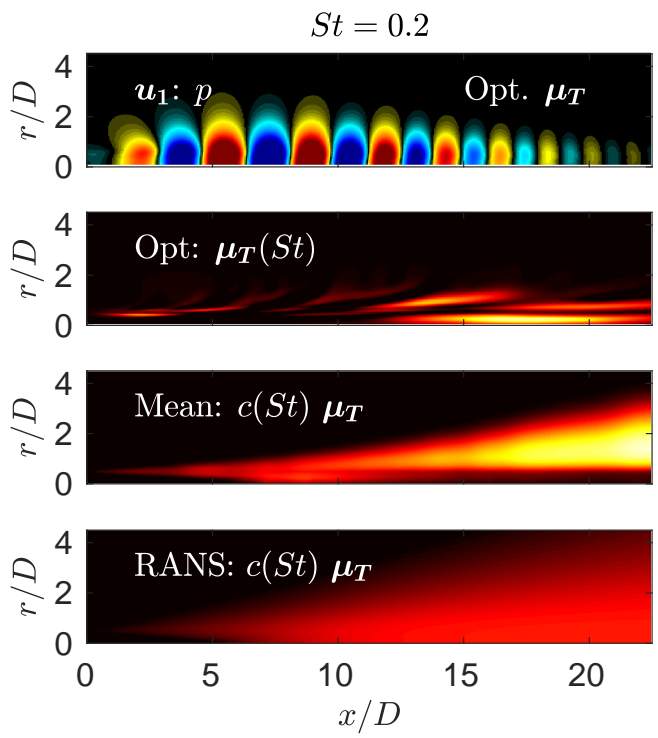
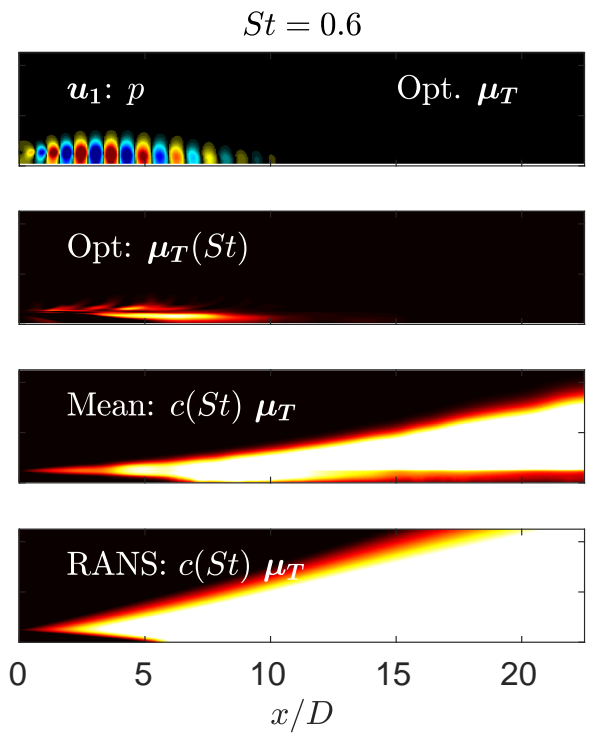

Figure 9: Comparisons of the optimal eddy-viscosity fields (i.e. full-field optimal, meanflow consistent, and RANS) and the associated dominant resolvent mode found via the optimization for $S t=0.2$ and 0.6. Contours for all six eddy-viscosity fields are set from 0 to $3 \times 10^{-3}$.

\subsection{Structure of the eddy-viscosity fields}

For the constant eddy viscosity, RANS-based, and mean-flow consistent eddy-viscosity fields, the optimization is over a single constant scaling amplitude value, whose optimal value is plotted as a function of frequency (still for $m=0$ ) in figure 8 (a,c,d) and the maximum value of the optimal field in (b). For all models, the frequency dependence of the values are similar, with three regions of interest: $S t=0.05-0.3, S t=0.3-0.8$ and St $>0.8$.

In the low frequency region, the baseline jet response comprises spatially extensive Orr-type modes that have a strong Reynolds number dependence, and they require a relatively larger eddy viscosity to damp them. For $S t=0.05$ the ratio of molecular Reynolds number to effective Reynolds number is $\mu_{j} / \boldsymbol{\mu}_{T} \approx 13,500$, for the constant model, a four order-of-magnitude difference when compared to the molecular viscosity.

In the middle frequency regime, where the baseline spectrum transitions from the broadband, viscous Orr mechanism to the low-rank, inviscid KH mechanism, much less eddy viscosity is needed, and we expect (confirming below, in $\S 7.1$ ) insensitivity to the overall value based on the relatively good alignment achieved in the baseline case. As frequency increases, the responses then transition back to a mix of $\mathrm{KH}$ and Orr-type waves, with a progression towards broadband, viscous Orr modes at higher frequency.

At these higher frequencies, we see that the low-frequency dependence on inverse effective Reynolds number resumes, similar to low the frequencies. Interestingly, this trend indicates that at higher frequencies $R e_{T} \rightarrow R e_{j}$ such that the effect of eddy viscosity "turns-off" as frequency increases and the associated wavepacket wavelength becomes small, approaching finer-scale turbulence, as would be expected on physical grounds.

For the full field eddy-viscosity optimization, we first recall that its primary purpose is to determine what may be an upper bound for how well any eddy-viscosity model 
could perform. Given that the alignments between the resolvent and SPOD modes were not significantly higher for the optimized scheme than for the modeled eddyviscosity approaches (with optimal parameters), the detailed eddy-viscosity fields are of lesser importance. Still, some aspects of the physics, such as the spatial locations where Reynolds stresses become important for each frequency, are apparent in the optimized fields. Figure 9 presents the optimized fields for two selected Strouhal numbers, comparing them to both the RANS and mean-flow consistent eddy-viscosity fields scaled by their optimal coefficient $c$ at each frequency. In addition, the dominant resolvent mode, computed with the displayed optimal-eddy-viscosity field, is shown for comparison with the eddy-viscosity fields. The contour for the eddy-viscosity fields are set from 0 to the maximum value of the $S t=0.6$ optimal eddy-viscosity field.

Overall, both frequencies present eddy-viscosity fields that are complex, unsurprising given the ability of the optimization to choose any eddy-viscosity field, constrained only by the structure of the equations and positivity. The optimization removes viscosity from the potential core, compared to the initial guess, approximately corresponding to the interior region of the jet relative to the critical layer, while increasing the turbulent viscosity just outside of the critical layer, and most strongly in the portion of the wavepacket where it decays downstream. When restricting the view to the region where the resolvent/SPOD mode has significant amplitude, there is a correspondence between the modeled eddy-viscosity fields and the optimal ones.

The optimal eddy-viscosity fields pinpoint the locations where linear structures begin to break down (i.e. where nonlinearities/Reynolds stresses become important) and inform what features an eddy-viscosity model must include. Through the above comparisons of figure 9, we can see that both the RANS and mean-flow consistent eddy-viscosity fields have such features directly embedded into their structure, explaining the ability of each model to achieve nearly optimal results. Additionally, we will show in the following section how such features also explain the ability of the RANS and mean-flow consistent models to predict the subdominant modes, which require further turbulence modeling downstream.

\section{Alignment of subdominant modes}

The optimization presented attempts to align only the dominant SPOD and resolvent modes. However, subdominant modes are also of interest, particularly as they are necessary to reconstruct flow statistics in the near field and are relevant for modeling coherence decay associated with 'jittering of wavepackets' to produce sound (Cavalieri et al. 2011). We are interested in two questions, whether alignment with only the dominant mode substantially alters the alignment of the subdominant modes and the effect of expanding the optimization to subdominant modes. The former case is assessed first using the optimal parameters for each method, the subdominant modes are computed and shown in figure 10 for modes 2 and 3 for the $S t=0.6, m=0$ frequency-wavenumber pair.

Starting with the second mode, compared to the baseline case $\left(R e_{T}=3 \times 10^{4}\right)$ all EVRA models result in significantly better alignments, reaching $\approx 70 \%$ for the RANS and mean-flow consistent models. We see that these are superior to the optimal eddyviscosity field, which is only fitted to align the dominant mode. The RANS and mean-field models are also superior for the third, and the fourth and fifth modes (not shown for brevity), but with an alignment that falls off with increasing mode number. This is largely attributable to the flattening of the SPOD spectrum and statistical limitations typically seen beyond the first few SPOD modes.

Assessing the subdominant modes returns us to the question of correlated forcing in 


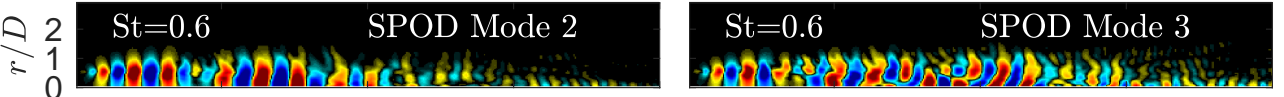

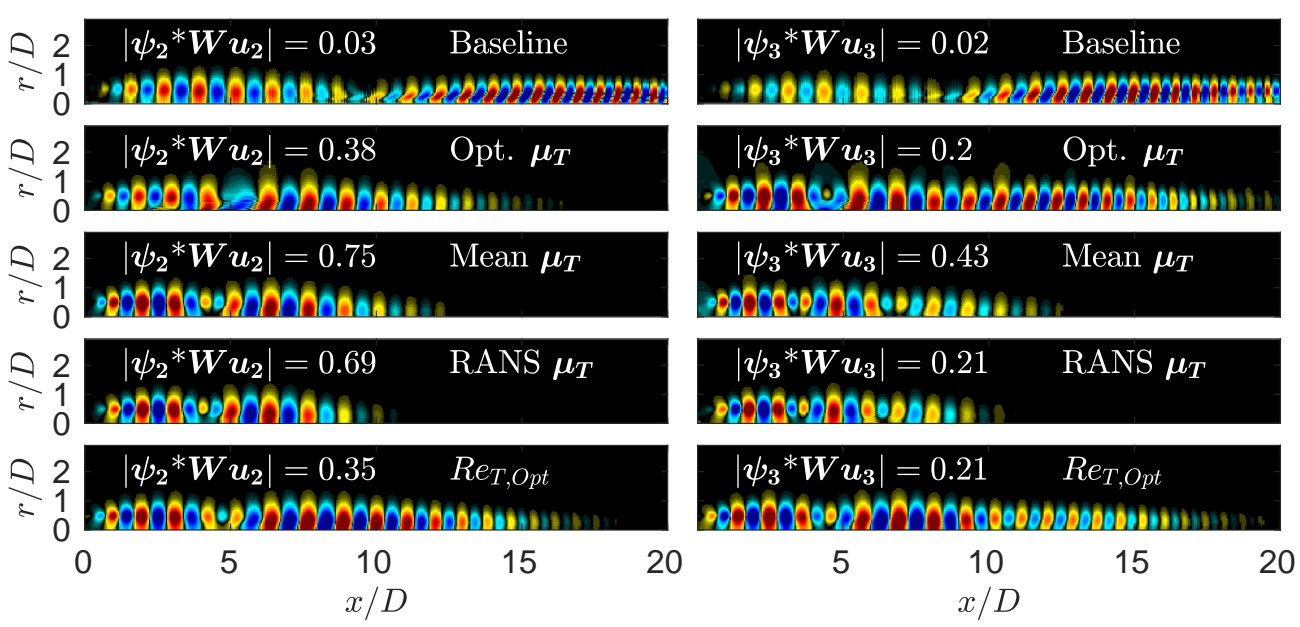

Figure 10: Subdominant modes 2 and 3 at $S t=0.6, m=0$ in the left and right columns respectively for SPOD, baseline, optimal eddy-viscosity field, mean-flow consistent model, RANS model, the turbulent Reynolds number.

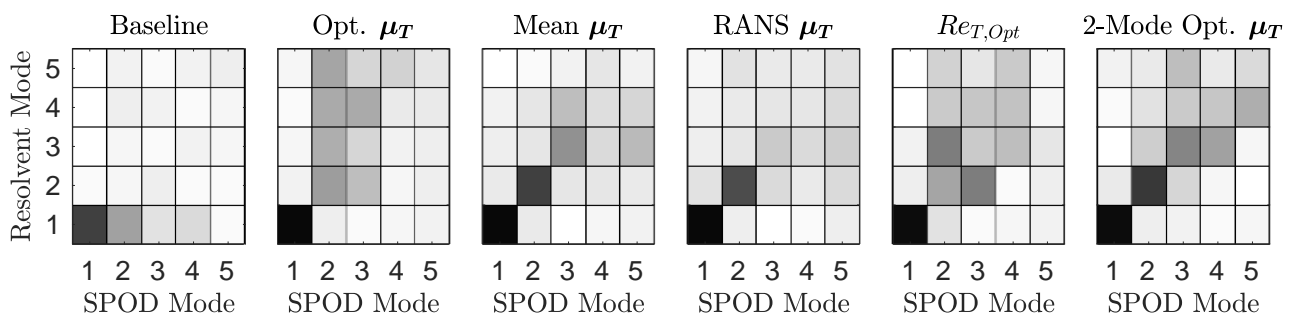

Figure 11: Projections of the first five SPOD modes into the first five resolvent modes computed for all eddy-viscosity resolvent models at $S t=0.6, m=0$, including the 2-mode optimization shown in figure 12.

turbulent flows. As already discussed, the CSD tensor of the response may be related to CSD tensor of the forcing through the resolvent analysis. However, the CSD tensor for the forcing is generally unknown. In this study, we instead assume the CSD tensor is uncorrelated in space and attempt to adjust the resolvent operator such that uncorrelated forcing suffices. To assess how well our optimization of the first SPOD mode performed with regards to diagonalizing the forcing CSD, the projections of five SPOD modes are compared with the first five modes from each eddy-viscosity method (including a 2mode optimization described next) in figure 11. The plots show that the EVRA models, particularly the RANS and mean-flow consistent models, are superior at diagonalizing the CSD as compared to the baseline case.

Although the optimal eddy-viscosity field, aligned only with the dominant SPOD mode, 

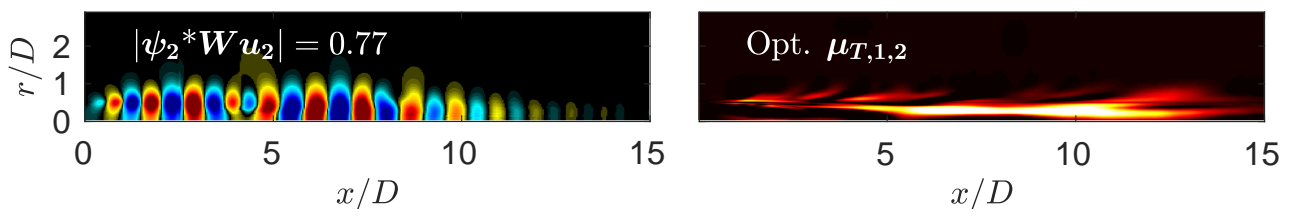

Figure 12: The second subdominant mode at $S t=0.6$ and the associated eddy-viscosity field that provides the optimal alignment for both modes. The contour for the eddyviscosity field is set to the same value as those shown in figure 9 from 0 to $3 \times 10^{-3}$.

shows improvements in the subdominant modes, the optimization can easily be extended to align subdominant modes. By definition, the optimal framework can find the overall eddy-viscosity field for any arbitrary number of modes and achieve alignment superior to any eddy-viscosity model. However, convergence issues with increasing SPOD mode number suggest that optimizing for many modes (e.g. $n>5$ ) would have marginal returns. For this study, only the optimization of both the first and second modes at $S t=0.6, m=0$ is presented to demonstrate the generality of the optimization framework and the physical implications of the associated eddy-viscosity field for the subdominant modes.

Figure 12 presents the aligned resolvent mode via the optimization and the associated eddy-viscosity field for the first subdominant mode. By including the second SPOD mode the optimization is able to achieve an alignment of $77 \%$, superior than any of the other eddy-viscosity models. Further, the alignment of the dominant mode, 96\%, is still maintained and interestingly, the remaining subdominant modes also increase their projections as shown in figure 11. The reasoning for the latter observation is due to the difference in mechanisms of the dominant and subdominant modes at $S t=0.6$, $m=0$. The dominant mode is KH-type, while the subdominant modes are of Orr-type. By aligning just the first Orr-type mode, the remaining Orr modes are also brought into greater alignment.

The increase in alignment is directly attributable to the additional modeled eddyviscosity. This can be seen by comparing the $\boldsymbol{\mu}_{T, 1,2}$ field to the KH-type only field, $\boldsymbol{\mu}_{T, 1}$, shown in figure 9 . The inclusion of the second mode presents further eddy-viscosity that acts further downstream and towards the centerline, representative of the Orr-mechanism at $m=0$ for turbulent jets (Pickering et al. 2019a). It is this additional downstream eddyviscosity, present in both the RANS and mean-flow consistent models, that is responsible for the increased subdominant mode alignment. Considering the simpler RANS (and mean-flow) model also shows similar downstream structure, we investigate its merit for a complete model in the next section.

\section{Towards a complete EVRA model for turbulent jets}

Through the previous sections we have shown that both the RANS and mean-flow consistent eddy-viscosity models perform well across Strouhal numbers from 0.05 to 1 at $m=0$, provided the overall constant associated with their application to the disturbance fields is optimized (at each frequency and azimuthal mode number). In this section, we consider the sensitivity of the results with respect to the choice of a constant, and show that over a range of frequencies and azimuthal mode numbers, the alignment is relatively insensitive to this constant, such that a single, universal value may be acceptable. While both RANS and mean-flow consistent models both performed well with optimal coefficients, we focus here exclusively on the RANS $k-\epsilon$ model as 


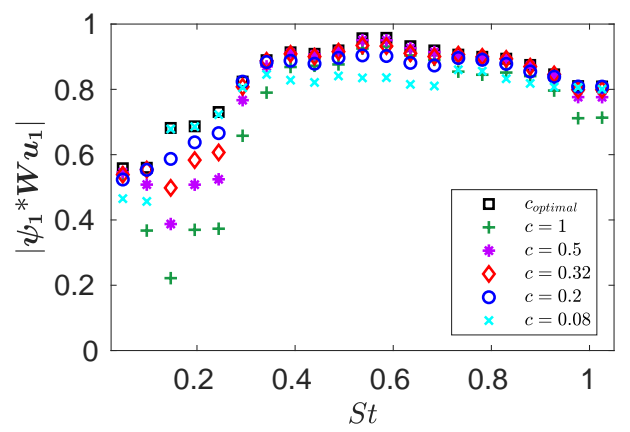

Figure 13: Alignments across all Strouhal numbers for the RANS eddy-viscosity model coefficients compared with the optimal RANS coefficient at each frequency. The RANS coefficients are $c=[1,0.5,0.32,0.2,0.08]$.

(a)

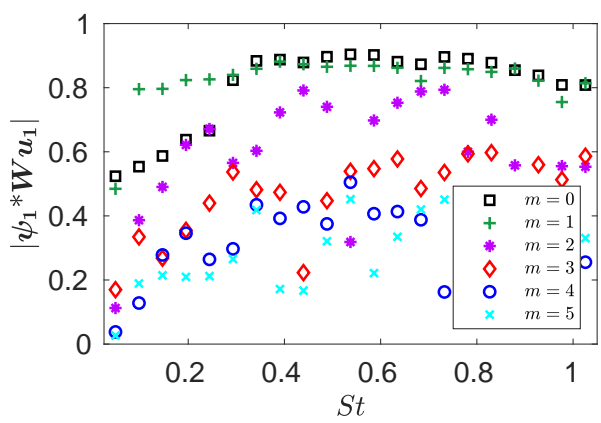

(b)

Baseline

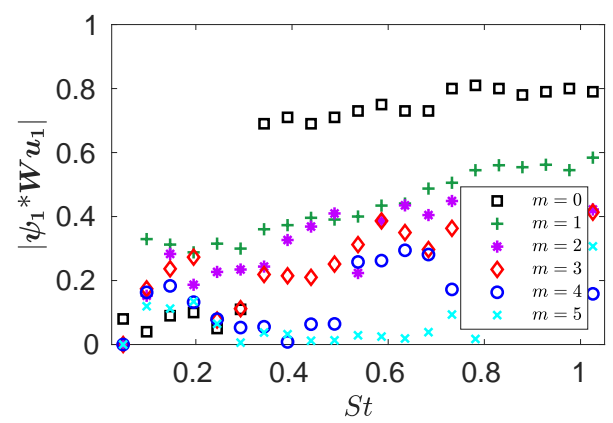

Figure 14: Alignments for frequencies, $S t \in[0.05,1]$, and azimuthal wavenumbers, $m \in$ $[0,5]$, for the (a) RANS eddy-viscosity model using $c=0.2$ and the (b) baseline, constant eddy-viscosity case (i.e. $\left.R e_{T}=3 \times 10^{4}\right)$.

it is better regarded as universal across a range of flows as compared to the specialized mean-flow consistent model. We then apply EVRA-RANS to the $M_{j}=0.4$ jet using the same constant to six azimuthal wavenumbers, $m \in[0,5]$, and find substantially improved predictions when compared to baseline predictions. Similar observations are made when using the same EVRA-RANS model for both the transonic and supersonic jets. Finally, we present the effect of the eddy-viscosity on the resolvent spectra.

\subsection{Frequency and azimuthal mode sensitivity}

As shown in figure 8, the optimal RANS coefficient ranged from $c=0.7-0.004$, with a constant region at mid-frequencies where $c=0.5$. The fully optimized eddy-viscosity field produced only marginally better alignment for most cases, and this suggests that the results may not be very sensitive to the precise constant. We tested the RANS model across a range of frequencies with proposed "universal" values of these constants of $c=[1,0.5,0.32,0.2,0.08]$. The resulting alignments are plotted versus frequency in figure 13. With little compromise compared to the optimal constant for each frequency, a single constant of $c=0.2$ leads to good alignment across all frequencies up to $S t=1$.

Considering now higher azimuthal modes, figure 14 (a) presents the alignment of the EVRA-RANS model with SPOD with $c=0.2$ for $m=0$ to 5 , while figure 14 (b) provides 
(a)

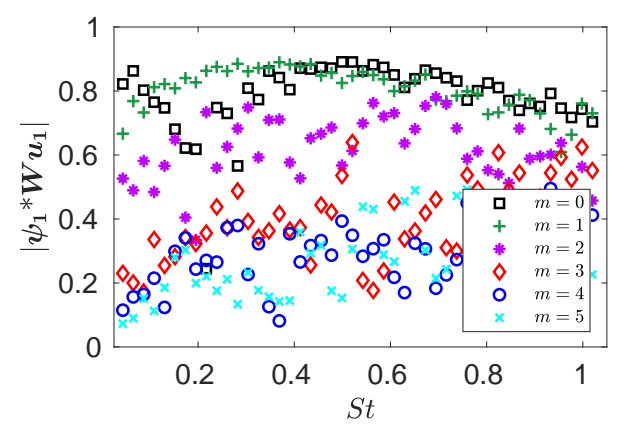

(b)

$$
M_{j}=1.5
$$

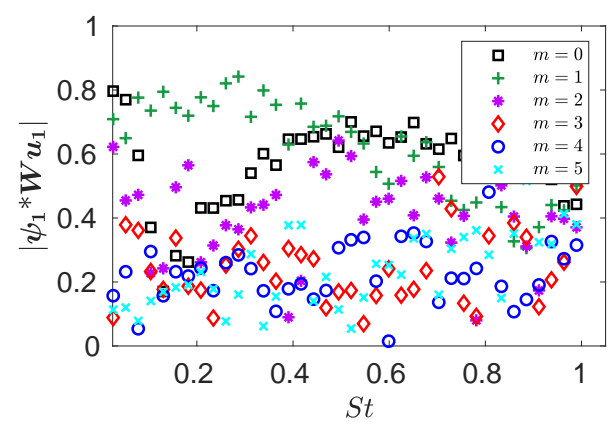

Figure 15: Alignments using the RANS eddy-viscosity model with coefficient $c=0.2$ across Strouhal numbers $S t \in[0.05,1]$ and azimuthal wavenumbers $m \in[0,5]$ for the (a) $M_{j}=0.9$ and (b) 1.5 jets.

the alignments for the baseline case. The EVRA-RANS model substantially increases the alignments for all nonzero wavenumbers. The results for $m=1$ are particularly encouraging, with a uniform $80 \%$ alignment across all frequencies. Azimuthal modes greater than 1 result in poorer alignment, albeit much improved compared to the baseline case, particularly when $m>2$. It should also be noted that energy drops off rapidly at these higher azimuthal mode numbers and, consequently, statistical errors (i.e. lack of convergence) exist in the SPOD modes.

For $m=1$, the ability of the eddy-viscosity resolvent models to represent low-frequency modes has implications for streaky structures arising from the lift-up mechanism observed in the global SPOD spectrum at these low frequencies by Pickering et al. (2019a). Their results also showed that resolving modes related to streaks in the resolvent spectrum required an eddy-viscosity model (using the TKE model reported by Pickering et al. (2019b) with $c=0.0065)$. The lift-up mechanism is known to present a rapid spatial growth of streamwise streaks until viscous dissipation becomes dominant and the structures decay (Hultgren \& Gustavsson 1981). In the case of turbulent jets, the spatial extent of resolvent modes increases as frequency decreases, as such, including an eddy-viscosity model becomes ever more important for resolvent modes computed at low-frequencies.

\subsection{Transonic and supersonic turbulent jets}

We now generalize the RANS-EVRA model performance for both $M_{j}=0.9$ and 1.5 turbulent jets. Figure 15 provides the alignments across frequencies and azimuthal wavenumbers for the transonic (a) and supersonic (b) jet. The transonic jet gives substantial agreement for $m=0$ and $m=1$ of about $80 \%$ for much of the frequency range, while $m=2$ shows alignments averaging at $60 \%$. For the supersonic jet, we do not see as promising alignments, however much improved from the $R e_{T}=3 \times 10^{4}$ alignments (not shown here).

A deeper investigation of the $M_{j}=1.5$ results finds that the dominant SPOD mode contains both $\mathrm{KH}$ and Orr-type mechanisms, while the resolvent modes separate each of these mechanisms. This is again due to a lack of eigenvalue separation in the SPOD spectra resulting in unconverged modes. Nevertheless, these results provide reasonable and, at the least, significantly improved projections across the most energetic frequencies and wavenumbers of subsonic, transonic, and supersonic regimes of turbulent jets using one RANS-based eddy-viscosity model. 


\section{4}
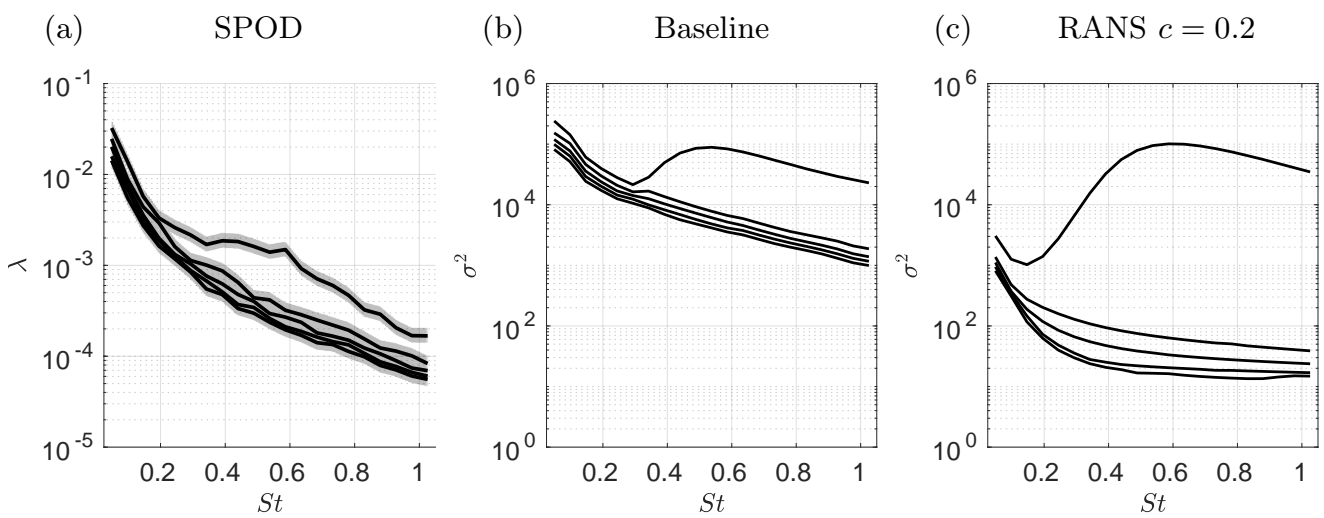

Figure 16: Spectra of first five (a) SPOD, (b) baseline resolvent, and (c) the RANS eddy-viscosity model resolvent modes at $m=0$ for $S t \in[0.05-1]$.

\subsection{Singular values}

After setting the eddy-viscosity model to a constant, we return to the $m=0$ case over $S t=0.05-1$ to assess the model's effect on the singular values and compare them to the baseline case and the SPOD eigenvalues. Figure 16 provides the spectra of the first five modes for SPOD (a) (accompanied by a shaded region providing the $95 \%$ confidence interval of the eigenvalues), the baseline resolvent model (b), and the RANS resolvent model (c) (using $c=0.2$ ) for $m=0$. Comparing the resolvent spectra to the SPOD spectra we immediately see that neither of the resolvent models present similar separation between $\lambda$ and $\sigma^{2}$. In fact, the RANS resolvent spectra has increased its energetic separation when compared to the baseline case.

We may link this behavior to multiple (in this case two) distinct mechanisms represented in the flow, the $\mathrm{KH}$ and Orr-mechanisms. When comparing the two resolvent models, the inclusion of an eddy-viscosity model presents a substantial effect on the amplification of the Orr modes, while the $\mathrm{KH}$ mechanism is relatively unchanged. The singular values related to the Orr mechanism decrease substantially, pulling away from the $\mathrm{KH}$ singular values, and also present a larger gain separation between adjacent Orr singular values. Schmidt et al. (2018) also observed this behavior at $S t=0.6, m=0$ when adjusting $R e_{T}$, finding that the squared singular values of the subdominant Orr modes were sensitive to the Reynolds number, scaling as $R e_{T}^{1.2}$. The same effect is observed here through the addition of the RANS eddy-viscosity model, interestingly (and perhaps unsurprising given the preceding discussions), figure 16 (c) provides similar values as those reported by Schmidt et al. (2018) at $S t=0.6, m=0$ using $R e_{T}=10^{3}$.

Figure 16 (a) and (c) also present implications for equation $(2.17 b)$. For a model with uncorrelated modes, if the SPOD and resolvent modes have been aligned then we have the relationship between the diagonal matrices, $\boldsymbol{\Lambda}=\boldsymbol{\Sigma} \boldsymbol{\Lambda}_{\boldsymbol{f f}} \boldsymbol{\Sigma}$, where $\boldsymbol{\Lambda}_{\boldsymbol{f f}}$ is a diagonal matrix containing the unknown forcing amplitude of the resolvent basis. The large differences in separation between the SPOD and RANS-EVRA spectra indicate that the KH and Orr mechanisms are forced by significantly different amplitudes. These amplitudes are contained in $\boldsymbol{\Lambda}_{\boldsymbol{f} \boldsymbol{f}}$, which can be directly computed from the known SPOD spectra, $\boldsymbol{\Lambda}_{\boldsymbol{f f}}=\boldsymbol{\Sigma}^{-1} \boldsymbol{\Lambda} \boldsymbol{\Sigma}^{-1}$. Such large differences in forcing amplitude can be explained by a recent sensitivity analysis by Pickering et al. (2019a) that determined the distinct spatial regions that lead to the most efficient amplification of the $\mathrm{KH}$, Orr, and the liftup mechanisms. They found that for the KH-type responses, the region is localized near 
the nozzle, while those for Orr-type responses are distributed throughout the domain, peaking multiple diameters downstream of the nozzle. Taking the turbulent kinetic energy field to be an estimate of perturbation sources in the flow, the nozzle activity is much smaller, by orders of magnitude, than the activity found downstream, thus the magnitude of the perturbations associated with each mechanism must be accounted for to construct a fully predictive model. Considering these observations, a logical next step in completing a resolvent-based turbulence model then lies in determining the forcing amplitudes that give an accurate reconstruction of the flow statistics.

\section{Conclusions}

Through a data-informed approach, we quantitatively tested the extent to which eddyviscosity models can improve the alignment between observed large-scale structures, educed via SPOD, and the dominant resolvent mode. We developed a novel, data-driven optimization framework to assess four different eddy-viscosity models and compare them to an optimal eddy-viscosity field that seeks the best alignment possible of any model.

The eddy-viscosity models chosen can be interpreted as a proxy for modeling the effect of the Reynolds stresses on large-scale structures. The Reynolds stresses may either be accounted for in the statistics of the nonlinear forcing CSD matrix or directly modeled in the linear operator (or both). Here we take the latter approach and find that regardless of the specific model, we obtain substantial improvements in alignment compared to a baseline case that used a constant eddy-viscosity model corresponding to a value of $R e_{T}=3 \times 10^{4}$. In particular, we find that models taking the form of traditional eddyviscosity models (e.g. RANS based), are superior to the use of an effective Reynolds number, and perform similarly as the optimal, best-case eddy-viscosity models.

Across the frequencies considered, the addition of eddy viscosity to the resolvent operator highlighted its effect on the different amplifications mechanisms namely, Orr-type, KH-type, and lift-up instabilities. The Orr-type mechanism was shown to be sensitive to the eddy-viscosity fields, whether at low or high frequencies as either the dominant mode or in the subdominant modes. The KH-type mode however is relatively insensitive to the eddy-viscosity field, a result expected from the inviscid nature of the inflectional KH instability. Subdominant modes, associated with the Orr-mechanism, were also assessed at $S t=0.6$ for the optimal parameters and gave significant improvements. All EVRA models provided subdominant modes that were more closely aligned with subdominant SPOD modes than in the baseline case. In particular, the models most similar to classic approaches, the RANS and mean-flow consistent models, presented the greatest diagonalization of the forcing CSD, providing evidence for each of the model's physical relevance.

We also showed the sensitivity of the RANS model to be rather weak, giving similar agreement for coefficients $c$ ranging over an order of magnitude. This demonstrates the robustness of using eddy-viscosity models for predictive EVRA, where SPOD modes are unavailable, as simply the inclusion of an eddy-viscosity model is more important than the exact magnitude of the model. Further, we observe that the coefficients of the RANS eddy-viscosity impart only a fraction of the computed eddy-viscosity field and suggest the use of coefficients $c$ ranging from 0.5 to 0.05 .

We also find promising performance when generalizing a frequency-independent RANS $(c=0.2)$ eddy-viscosity model across six azimuthal frequencies and three turbulent jets, spanning subsonic, transonic, and supersonic regimes. For the first three azimuthal wavenumbers (i.e. $m \in[0-2]$ ) we find substantially increased alignments among all three turbulent jets and across Strouhal numbers $S t \in[0.05,1]$. These results indicate 
that including an eddy-viscosity model, in particular a classical eddy-viscosity model, such as RANS or a mean-flow consistent model, aids in estimating the impact of the Reynolds stresses for resolvent analysis and we suggest implementation of either model in future EVRA models for increased model accuracy.

\section{Acknowledgments}

This research was supported by a grant from the Office of Naval Research (grant No. N00014-16-1-2445) with Dr. Steven Martens as program manager. E.P. was supported by the Department of Defense (DoD) through the National Defense Science \& Engineering Graduate Fellowship (NDSEG) Program. The LES study was performed at Cascade Technologies, with support from ONR and NAVAIR SBIR project, under the supervision of Dr. John T. Spyropoulos. The main LES calculations were carried out on DoD HPC systems in ERDC DSRC.

\section{Declaration of interests}

The authors report no conflict of interest.

\section{Appendix A. Linear damping term}

In addition to the studied eddy-viscosity models, we also investigated the impact of a linear damping term, which is equivalent to finite-time-horizon resolvent analysis introduced by Jovanović (2004). A recent resolvent study by Yeh \& Taira (2019) used linear damping to localize the forcing and response modes of an airfoil. Without the addition of a linear damping term, the forcing modes in their study extend infinitely in the upstream direction, and in the downstream direction for response modes. However, in real flows there exist significant short term (i.e. finite-time) dynamics of interest, such as intermittent behavior and development times for flow structures. A similar argument can be made with jet flows, where wavepackets may not be adequately bounded in the axial direction when compared to their corresponding SPOD modes, which by construction accurately account for the intermittent behavior and development times of structures in real flows.

For this model, the operator is modified so that

$$
\boldsymbol{L}_{\beta}=\boldsymbol{L}-\beta \boldsymbol{I} .
$$

where $\beta=1 / \tau>0$, and $\tau$ is the desired temporal decay rate. We used a line search to select, as a function of frequency, the value of $\beta$ that best aligns the dominant resolvent and SPOD modes. The optimization (at each frequency) is with respect to a single constant $\beta$. For this optimization an initial condition of $\beta=0$ was used. The sensitivity of the optimal $\beta$ to this initial guess was checked for selected frequencies and verified that (at least for $\beta>0$ ), a global maximum was achieved.

Figure 17 presents the increased alignments for the linear damping case. Although the linear damping is able to increase projection coefficients, the performance is significantly inferior to the eddy-viscosity models presented in the body of the text, likely due to its monolithic damping effect over all wavenumbers, whereas the eddy-viscosity methods directly address the affect of the Reynolds stresses. Overall, the linear damping model, while an improvement over the baseline case, is generally inferior to the eddy-viscosity models, and was only considered for the $M_{j}=0.4, m=0$, and $S t \in[0.05,1]$ cases. 


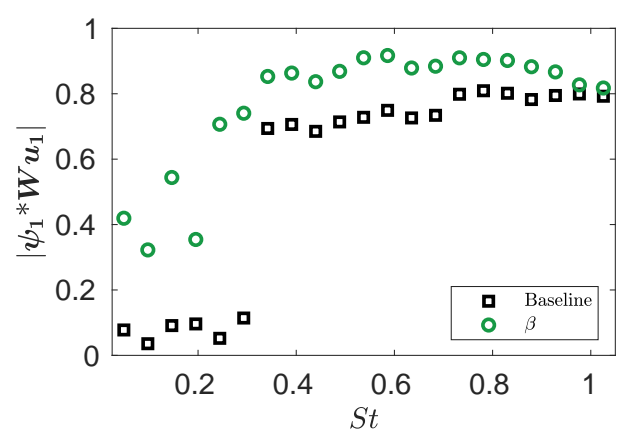

Figure 17: Optimal alignments for the linear damping term and the baseline case, $R e_{T}=$ $3 \times 10^{4}$.

\section{Appendix B. Navier-Stokes equations}

Below are the continuous equations for continuity, momentum, and energy, respectively, in vector form and used for the resolvent analysis presented. To construct the operators used, all equations are transformed azimuthally, undergo a standard Reynolds decomposition (keeping on linear terms for the linear operator), and discretized in the radial and streamwise directions. All terms associated with the left-hand side of the equations are used to construct $\boldsymbol{L}$, while all terms on the right-hand side, associated with an eddy-viscosity term, are used to construct the operator $\boldsymbol{L}_{T}$,

$$
\begin{aligned}
& i \omega \rho+(\boldsymbol{u} \cdot \nabla) \rho+\rho \Theta \\
& =0 \\
& i \omega \boldsymbol{u}+(\boldsymbol{u} \cdot \nabla) \boldsymbol{u}+\frac{1}{\gamma M_{j}^{2}} \nabla(\rho T)-\frac{1}{R e_{j}}\left(\nabla^{2} \boldsymbol{u}+(1+\lambda) \nabla \Theta\right) \\
& =\nabla \cdot\left[\mu_{T}\left((\nabla \boldsymbol{u})+(\nabla \boldsymbol{u})^{T}-\frac{2}{3} \Theta \mathbb{I}\right)\right] \\
& \rho\left(i \omega T+(\boldsymbol{u} \cdot \nabla) T+\frac{1}{\gamma M_{j}^{2}} T \Theta\right)-\frac{1}{(\gamma-1) M_{j}^{2} R e_{j} \operatorname{Pr}_{\infty}} \nabla^{2} T \\
& -\frac{\gamma M^{2}}{R e_{j}}\left[\frac{1}{2}\left\{(\nabla \boldsymbol{u})+(\nabla \boldsymbol{u})^{T}\right\}:\left\{(\nabla \boldsymbol{u})+(\nabla \boldsymbol{u})^{T}\right\}-\frac{2}{3} \Theta^{2}\right] \\
& =\frac{\boldsymbol{\mu}_{T}}{(\gamma-1) M_{j}^{2} P r_{\infty}} \nabla^{2} T+\gamma M_{j}^{2} \mu_{T}\left[\frac{1}{2}\left\{(\nabla \boldsymbol{u})+(\nabla \boldsymbol{u})^{T}\right\}:\left\{(\nabla \boldsymbol{u})+(\nabla \boldsymbol{u})^{T}\right\}-\frac{2}{3} \Theta^{2}\right] \text {. }
\end{aligned}
$$

\section{REFERENCES}

Brès, G. A., Ham, F. E., Nichols, J. W. \& Lele, S. K. 2017 Unstructured large-eddy simulations of supersonic jets. AIAA Journal pp. 1164-1184.

Brès, G. A., Jordan, P., Jaunet, V., Le Rallic, M., Cavalieri, A. V. G., Towne, A., Lele, S. K., Colonius, T. \& Schmidt, O. T. 2018 Importance of the nozzle-exit boundary-layer state in subsonic turbulent jets. J. Fluid Mech. 851, 83-124.

Butler, K. M. \& FArrell, B. F. 1992 Three-dimensional optimal perturbations in viscous shear flow. Physics of Fluids A: Fluid Dynamics 4 (8), 1637-1650. 
Cavalieri, A. V. G., Jordan, P., Agarwal, A. \& Gervais, Y. 2011 Jittering wave-packet models for subsonic jet noise. Journal of Sound and Vibration 330 (18-19), 4474-4492.

Cavalieri, A. V. G., Rodríguez, D., Jordan, P., Colonius, T. \& Gervais, Y. 2013 Wavepackets in the velocity field of turbulent jets. J. Fluid Mech. 730, 559-592.

Cess, R. D. 1958 A survey of the literature on heat transfer in turbulent tube flow. Tech. Rep. pp. 8-0529-R24.

Chu, B.-T. 1965 On the energy transfer to small disturbances in fluid flow (Part I). Acta Mechanica 1 (3), 215-234.

Cossu, C., Pujals, G. \& Depardon, S. 2009 Optimal transient growth and very large-scale structures in turbulent boundary layers. J. Fluid Mech. 619, 79-94.

Crighton, D. G. \& Gaster, M. 1976 Stability of slowly diverging jet flow. J. Fluid Mech. 77 (2), 397-413.

Crouch, J. D., Garbaruk, A. \& Magidov, D. 2007 Predicting the onset of flow unsteadiness based on global instability. Journal of Computational Physics 224 (2), 924-940.

Del Alamo, J. C. \& Jimenez, J. 2006 Linear energy amplification in turbulent channels. J. Fluid Mech. 559, 205-213.

Deprés, DAVID 2003 Analyse physique et modélisation des instationnarités dans les écoulements d'arrière-corps transsoniques. $\mathrm{PhD}$ thesis, Aix-Marseille 2.

Duraisamy, K., Iaccarino, G. \& XiaO, H. 2019 Turbulence modeling in the age of data. Ann. Rev. Fluid Mech. $\mathbf{5 1 .}$.

Farrell, B. F. \& IoAnnou, P. J. 1993 Optimal excitation of three-dimensional perturbations in viscous constant shear flow. Physics of Fluids A: Fluid Dynamics 5 (6), 1390-1400.

Foures, Dimitry PG, Dovetta, Nicolas, Sipp, Denis \& Schmid, Peter J 2014 A dataassimilation method for reynolds-averaged navier-stokes-driven mean flow reconstruction. Journal of fluid mechanics 759, 404-431.

Garnaud, X., Lesshafft, L., Schmid, P. J. \& Huerre, P. 2013 The preferred mode of incompressible jets: linear frequency response analysis. J. Fluid Mech. 716, 189-202.

Georgiadis, N. J., Yoder, D. A. \& Engblom, W. A. 2006 Evaluation of modified twoequation turbulence models for jet flow predictions. AIAA journal 44 (12), 3107-3114.

Hansen, P. C. \& O'Leary, D. P. 1993 The Use of the L-Curve in the Regularization of Discrete Ill-Posed Problems. SIAM Journal on Scientific Computing 14 (6), 1487-1503.

HoARau, YANnick 2002 Analyse physique par simulation numérique et modélisation des écoulements décollés instationnaires autour de surfaces portantes. $\mathrm{PhD}$ thesis, Toulouse, INPT.

Hoarau, Yannick, Faghani, D, Braza, Marianna, Perrin, Rodolphe, Anne-Archard, Dominique \& Ruiz, D 2003 Direct numerical simulation of the three-dimensional transition to turbulence in the incompressible flow around a wing. Flow, turbulence and combustion 71 (1-4), 119-132.

Hultgren, L. S. \& Gustavsson, L. H. 1981 Algebraic growth of disturbances in a laminar boundary layer. The Physics of Fluids 24 (6), 1000-1004.

Hwang, Y. 2016 Mesolayer of attached eddies in turbulent channel flow. Physical Review Fluids $1(6), 064401$.

Hwang, Y. \& Cossu, C. $2010 a$ Amplification of coherent streaks in the turbulent Couette flow: an input-output analysis at low Reynolds number. J. Fluid Mech. 643, 333-348.

Hwang, Y. \& Cossu, C. $2010 b$ Linear non-normal energy amplification of harmonic and stochastic forcing in the turbulent channel flow. J. Fluid Mech. 664, 51-73.

Illingworth, S. J., Monty, J. P. \& Marusic, I. 2018 Estimating large-scale structures in wall turbulence using linear models. J. Fluid Mech. 842, 146-162.

Jaunet, V., Jordan, P. \& Cavalieri, A. V. G. 2017 Two-point coherence of wave packets in turbulent jets. Physical Review Fluids 2 (2), 024604.

Jeun, J., Nichols, J. W. \& Jovanović, M. R. 2016 Input-output analysis of high-speed axisymmetric isothermal jet noise. Phys. Fluids 28 (4), 047101.

Jovanović, M. R. 2004 Modeling, analysis, and control of spatially distributed systems. University of California at Santa Barbara, Dept. of Mechanical Engineering.

Jovanović, M. R. \& BAmieh, B. 2005 Componentwise energy amplification in channel flows. J. Fluid Mech. 534, 145-183.

Lesshafft, L., Semeraro, O., Jaunet, V., Cavalieri, A. V. G. \& Jordan, P. 2019 
Resolvent-based modelling of coherent wavepackets in a turbulent jet. Phys. Rev. Fluids $4(6), 063901$.

Lumley, J. L. 1967 The structure of inhomogeneous turbulent flows. Atmospheric turbulence and radio propagation pp. $166-178$.

Lumley, J. L. 1970 Stochastic tools in turbulence. J. Fluid Mech. 67, 413-415.

Malkus, W. V. R. 1956 Outline of a theory of turbulent shear flow. J. Fluid Mech. 1 (5), 521-539.

Mattsson, K. \& Nordström, J. 2004 Summation by parts operators for finite difference approximations of second derivatives. J. Computat. Phys. 199 (2), 503-540.

Maulik, R., San, O., Jacob, J.D. \& CRICK, Ch. 2019 Sub-grid scale model classification and blending through deep learning. J. Fluid Mech. 870.

McKeon, B. J. \& Sharma, A. S. 2010 A critical-layer framework for turbulent pipe flow. J. Fluid Mech. 658, 336-382.

Meliga, P., Pujals, G. \& Serre, E. 2012 Sensitivity of 2-D turbulent flow past a D-shaped cylinder using global stability. Physics of Fluids 24 (6), 061701.

Metтot, C., Sipp, D. \& BÉzard, H. 2014 Quasi-laminar stability and sensitivity analyses for turbulent flows: prediction of low-frequency unsteadiness and passive control. Physics of Fluids 26 (4), 061701.

Michalke, A. 1971 Instability of a compressible circular free jet with consideration of the influence of the jet boundary layer thickness. Z. für Flugwissenschaften 19 (8), 319-328.

Moarref, R. \& Jovanović, M. R. 2012 Model-based design of transverse wall oscillations for turbulent drag reduction. J. Fluid Mech. 707, 205-240.

Moarref, R., Sharma, A. S., Tropp, J. A. \& McKeon, B. J. 2013 Model-based scaling of the streamwise energy density in high-Reynolds-number turbulent channels. J. Fluid Mech. 734, 275-316.

Mohseni, K. \& Colonius, T. 2000 Numerical treatment of polar coordinate singularities. J. Computat. Phys. 157 (2), 787-795.

Morra, P., Semeraro, O., Henningson, D. S. \& Cossu, C. 2019 On the relevance of Reynolds stresses in resolvent analyses of turbulent wall-bounded flows. J. Fluid Mech. 867, 969-984.

Nogueira, P. A. S., Cavalieri, A. V. G., Jordan, P. \& Jaunet, V. 2019 Large-scale, streaky structures in turbulent jets. J. Fluid Mech. 873, 211-237.

Oberleithner, K., Paschereit, C. O. \& Wygnanski, I. 2014 On the impact of swirl on the growth of coherent structures. J. Fluid Mech. 741, 156-199.

Parish, E. \& Duraisamy, K. 2016 A paradigm for data-driven predictive modeling using field inversion and machine learning. Journal of Computational Physics 305, 758-774.

Pickering, E., Rigas, G., Nogueira, P. A. S., Cavalieri, A. V. G., Schmidt, O. T. \& Colonius, T. $2019 a$ Lift-up, Kelvin-Helmholtz and Orr mechanisms in turbulent jets. arXiv preprint arXiv:1909.09737 .

Pickering, E., Rigas, G., Sipp, D., Schmidt, O. T. \& Colonius, T. $2019 b$ Eddy viscosity for resolvent-based jet noise models. In 25th AIAA/CEAS Aeroacoustics Conference, p. 2454.

Pickering, E., Towne, A., Jordan, P. \& Colonius, T. 2020 Resolvent-based jet noise models: a projection approach. In AIAA Scitech 2020 Forum, p. 0999.

Pope, S. B. 1978 An explanation of the turbulent round-jet/plane-jet anomaly. AIAA journal 16 (3), 279-281.

Pujals, G., García-Villalba, M., Cossu, C. \& Depardon, S. 2009 A note on optimal transient growth in turbulent channel flows. Physics of Fluids 21 (1), 015109.

Reynolds, W. C. \& Hussain, A. K. M. F. 1972 The mechanics of an organized wave in turbulent shear flow. Part 3. Theoretical models and comparisons with experiments. $J$. Fluid Mech. 54 (2), 263-288.

Reynolds, W. C. \& Tiederman, W. G. 1967 Stability of turbulent channel flow, with application to Malkus's theory. J. Fluid Mech. 27 (2), 253-272.

Rukes, Lothar, Paschereit, Christian Oliver \& Oberleithner, Kilian 2016 An assessment of turbulence models for linear hydrodynamic stability analysis of strongly swirling jets. European Journal of Mechanics-B/Fluids 59, 205-218. 
Sarkar, S., Erlebacher, G., Hussaini, M. Y. \& Kreiss, H. Otto. 1991 The analysis and modelling of dilatational terms in compressible turbulence. J. Fluid Mech. 227, 473-493.

Sartor, F., Mеттот, C. \& Sipp, D. 2014 Stability, receptivity, and sensitivity analyses of buffeting transonic flow over a profile. AIAA Journal 53 (7), 1980-1993.

Sasaki, K., Cavalieri, A. V. G., Jordan, P., Schmidt, O. T., Colonius, T. \& Brès, G. A. 2017 High-frequency wavepackets in turbulent jets. J. Fluid Mech. 830.

Schmid, P. J., Henningson, D. S. \& Jankowski, D. F. 2002 Stability and transition in shear flows. Appl. Mech. Rev. 55 (3), B57-B59.

Schmidt, O. T., Towne, A., Rigas, G., Colonius, T. \& Brès, G. A. 2018 Spectral analysis of jet turbulence. J. Fluid Mech. 855, 953-982.

Semeraro, O., Jaunet, V., Jordan, P., Cavalieri, A. V. \& Lesshafft, L. 2016 Stochastic and harmonic optimal forcing in subsonic jets. In 22nd AIAA/CEAS Aeroacoustics Conference, p. 2935.

Sharma, A. S. \& McKeon, B. J. 2013 On coherent structure in wall turbulence. J. Fluid Mech. 728, 196-238.

Tammisola, O. \& Juniper, M. P. 2016 Coherent structures in a swirl injector at $R e=4800$ by nonlinear simulations and linear global modes. J. Fluid Mech. 792, 620-657.

Thies, A. T. \& TAм, C. K. W. 1996 Computation of turbulent axisymmetric and nonaxisymmetric jet flows using the $k-\epsilon$ model. AIAA journal 34 (2), 309-316.

Towne, A., Colonius, T., Jordan, P., Cavalieri, A. V. \& Bres, G. A. 2015 Stochastic and nonlinear forcing of wavepackets in a mach 0.9 jet. In 21st AIAA/CEAS Aeroacoustics Conference, p. 2217.

Towne, A., Lozano-Durán, A. \& Yang, X. 2020 Resolvent-based estimation of space-time flow statistics. J. Fluid Mech. $\mathbf{8 8 3}$.

Towne, A., Schmidt, O. T. \& Colonius, T. 2018 Spectral proper orthogonal decomposition and its relationship to dynamic mode decomposition and resolvent analysis. J. Fluid Mech. $\mathbf{8 4 7}, 821-867$.

Trefethen, L. N., Trefethen, A. E., Reddy, S. C. \& Driscoll, T. A. 1993 Hydrodynamic stability without eigenvalues. Science 261 (5121), 578-584.

Vadarevu, S. B., Symon, S., Illingworth, S. J. \& Marusic, I. 2019 Coherent structures in the linearized impulse response of turbulent channel flow. J. Fluid Mech. 863, 1190-1203.

WANG, Z., LuO, K., Li, D., TAN, J. \& FAN, J. 2018 Investigations of data-driven closure for subgrid-scale stress in large-eddy simulation. Phys. Fluids 30, 125101.

YeH, C.-A. \& TAIRA, K. 2019 Resolvent-analysis-based design of airfoil separation control. J. Fluid Mech. 867, 572-610.

Yim, E., Meliga, P. \& Gallaire, F. 2019 Self-consistent triple decomposition of the turbulent flow over a backward-facing step under finite amplitude harmonic forcing. Proceedings of the Royal Society A 475 (2225), 20190018.

Zare, A., Jovanović, M. R. \& Georgiou, T. T. 2017 Colour of turbulence. J. Fluid Mech. 812, 636-680. 